J. Nonlinear Sci. Vol. 16: pp. 435-481 (2006)

DOI: $10.1007 / \mathrm{s} 00332-005-0733-2$

\title{
Nonlinear
} Science

(C) 2006 Springer Science+Business Media,Inc.

\section{On the Whitham Equations for the Defocusing Nonlinear Schrödinger Equation with Step Initial Data}

\author{
G. Biondini ${ }^{1}$ and Y. Kodama ${ }^{2}$ \\ 1 State University of New York at Buffalo, Department of Mathematics, Buffalo, NY 14260 \\ 2 Ohio State University, Department of Mathematics, Columbus, OH 43210
}

Received August 29, 2005; revised manuscript accepted for publication March 3, 2006

Online publication June 29, 2006

Communicated by T. Fokas

Summary. The behavior of solutions of the finite-genus Whitham equations for the weak dispersion limit of the defocusing nonlinear Schrödinger equation is investigated analytically and numerically for piecewise-constant initial data. In particular, the dynamics of constant-amplitude initial conditions with one or more frequency jumps (i.e., piecewise linear phase) are considered. It is shown analytically and numerically that, for finite times, regions of arbitrarily high genus can be produced; asymptotically with time, however, the solution can be divided into expanding regions which are either of genus-zero, genus-one, or genus-two type, their precise arrangement depending on the specifics of the initial datum given. This behavior should be compared to that of the Korteweg-de Vries equation, where the solution is divided into regions which are either genus-zero or genus-one asymptotically. Finally, the potential application of these results to the generation of short optical pulses is discussed: The method proposed takes advantage of nonlinear compression via appropriate frequency modulation, and allows control of both the pulse amplitude and its width, as well as the distance along the fiber at which the pulse is produced.

Key words. Nonlinear Schrödinger equation, weak dispersion limit, Whitham equations, dispersive shocks, dispersive regularizations, nonlinear optical pulses

MSC numbers. 35B25, 35Q55, 37K10, 37K40

The weak dispersion limit of the defocusing nonlinear Schrödinger (NLS) equation has been extensively studied in recent years (see, e.g., [15], [24], [29], [31], [33]), and in some sense it is well-characterized mathematically. Not as much is known, however, about the detailed behavior of the solutions for specific choices of initial datum [11], [12], [16], 
[27]. The purpose of this work is to present analytical and numerical results regarding the behavior of a special class of solutions of the NLS equation in the weak dispersion limit. More precisely, we consider the initial value problem for the NLS equation in the weak dispersion limit with the initial data having a constant-amplitude and piecewise-constant frequency. We believe that, on the one hand, this behavior is interesting mathematically, and, on the other hand, it could have potential applications in the generation of intense, ultra-short optical pulses. As such, it is worthy of further study.

The structure of this document is as follows: In Section 1 we introduce the problem, and in Section 2 we review some well-known results regarding the weak dispersion limit of the NLS equation. In Section 3 we discuss the behavior of solutions corresponding to "single-jump" initial conditions, which are the starting point for our investigation. Then, in Sections 4 and 5 we present the analytical calculations which are the main results of this work. In Section 6 we demonstrate these results through numerical simulations of the NLS equation and obtain further information about the solution behavior, and in Section 7 we discuss the application of our results to the generation of intense, ultra-short optical pulses. Appendix A.1 describes our nondimensionalizations and our choice of units, Appendices A.2 and A.3 review some known results regarding genus-one (i.e., periodic) solutions of the NLS equation, the Whitham averaging method, and the NLSWhitham equations, and Appendix A.4 gives the details of some calculations whose results are presented in Sections 3 and 4.

\section{The NLS Equation with Small Dispersion}

In this section we recall some basic results regarding the behavior of solutions of the nonlinear Schrödinger (NLS) equation with small dispersion. This will establish the background and the notation necessary to extend these results in the following sections.

The semiclassical limit of the NLS equation. We start from the defocusing NLS equation (that is, the NLS in the normal dispersion regime in optical fibers) in dimensionless form:

$$
i \epsilon \frac{\partial q}{\partial t}-\frac{1}{2} \epsilon^{2} \frac{\partial^{2} q}{\partial x^{2}}+|q|^{2} q=0,
$$

where we assume $0<\epsilon \ll 1$. In the context of optical fibers, $t$ represents the dimensionless propagation distance and $x$ is the dimensionless retarded time (cf. Appendix A.1). To study the weak dispersion limit of the NLS eq. (1.1), we first express the field $q(x, t)$ in a WKB form as

$$
q(x, t)=\sqrt{\rho(x, t)} \exp [i \varphi(x, t) / \epsilon],
$$

where $\rho(x, t)=|q(x, t)|^{2}$ and $\varphi(x, t)=\epsilon \arg q(x, t)=(i \epsilon / 2) \ln \left[q^{*}(x, t) / q(x, t)\right]$ represent respectively the local intensity and the normalized local phase of $q(x, t)$. We then introduce the normalized phase gradient

$$
u(x, t)=\partial \varphi(x, t) / \partial x,
$$

that is, $u(x, t)=(i \epsilon / 2)\left(q_{x}^{*}(x, t) q(x, t)-q^{*}(x, t) q_{x}(x, t)\right) /|q(x, t)|^{2}$. Throughout this work we refer to $x$ and $t$ as the space and time variables, respectively. By analogy with 
the fiber optics context, however, we will refer to $u(x, t)$ as the local frequency of the solution. With this decomposition, the NLS eq. (1.1) can be written in the form of a conservation law:

$$
\begin{aligned}
\frac{\partial \rho}{\partial t} & =\frac{\partial(\rho u)}{\partial x} \\
\frac{\partial(\rho u)}{\partial t} & =\frac{\partial}{\partial x}\left(\rho u^{2}+\frac{1}{2} \rho^{2}-\frac{1}{4} \epsilon^{2} \rho \frac{\partial^{2}}{\partial x^{2}} \ln \rho\right) .
\end{aligned}
$$

The weak dispersion limit of the defocusing NLS equation is defined as the problem of studying the solutions of (1.1) as $\epsilon \rightarrow 0^{+}$with initial condition expressed in terms of (1.2). The limit is singular, and it should be considered in the weak sense.

There are two main situations where the weak dispersion limit of the NLS equation is relevant: nonlinear fiber optics and Bose-Einstein condensation (BEC). In the context of nonlinear fiber optics, the weak dispersion limit is relevant for the long-distance transmission of non-return-to-zero (NRZ) pulses, as discussed in [27], or for the generation of intense short optical pulses, as proposed in this work. In the context of BEC, the semiclassical limit applies due to the very small value of Planck's constant $\hbar$ relative to quantities associated with macroscopic objects, i.e., $\hbar=\epsilon$. Normalizations appropriate for long-distance optical fiber communications were discussed in [16], whereas in Appendix A.1, we discuss scalings and nondimensionalizations relevant for the generation of intense short optical pulses. We emphasize, however, that the results presented in this work apply equally well to Bose-Einstein condensates and to other physical contexts where the NLS equation is relevant, such as for example ferromagnetics and water waves.

Hydrodynamic analogy and dam-breaking problem. If both $\rho$ and $u$ are smooth and $\rho>0$, and if $\epsilon \ll 1,(1.4)$ are approximated to leading order by the following reduced hydrodynamical system [28], [36]:

$$
\frac{\partial}{\partial t}\left(\begin{array}{l}
\rho \\
u
\end{array}\right)=\left(\begin{array}{ll}
u & \rho \\
1 & u
\end{array}\right) \frac{\partial}{\partial x}\left(\begin{array}{l}
\rho \\
u
\end{array}\right) .
$$

Equation (1.5) is called the dispersionless NLS equaton, and is used to describe a surface wave motion in shallow water. In the hydrodynamical setting, $\rho$ and $-u$ represent respectively the depth and velocity of water, and $x$ and $t$ are dimensionless space and time. For $\rho>0$, the eigenvalues $u \pm \sqrt{\rho}$ of the coefficient matrix are real, and the system (1.5) is strictly hyperbolic. This system, which is known as the shallow water wave equation and has been intensively studied (see e.g. [36]), can be rewritten in Riemann invariant (i.e., diagonal) form as

$$
\frac{\partial r_{k}}{\partial t}=s_{k} \frac{\partial r_{k}}{\partial x}, \quad k=1,2
$$

where the Riemann invariants $r_{1,2}(x, t)$ are given by

$$
r_{1}=u-2 \sqrt{\rho}, \quad r_{2}=u+2 \sqrt{\rho},
$$

and the characteristic speeds $s_{1,2}(x, t)$ are

$$
s_{1}=\frac{1}{4}\left(3 r_{1}+r_{2}\right)=u-\sqrt{\rho}, \quad s_{2}=\frac{1}{4}\left(r_{1}+3 r_{2}\right)=u+\sqrt{\rho} .
$$


Note that $s_{k}>0$ implies a left-moving wave (i.e., $d x / d t=-s_{k}$ ), and that (1.7) are equivalent to

$$
\rho=\frac{1}{16}\left(r_{2}-r_{1}\right)^{2}, \quad u=\frac{1}{2}\left(r_{1}+r_{2}\right) .
$$

Since the system of PDEs described by (1.6) is strictly hyperbolic, it is possible to show that, for "rarefaction" initial data, namely, when $r_{1,2}(x, 0)$ are both monotonically decreasing functions of $x$, a global solution exists for all $t>0$. In many cases of interest, however, the initial data do not satisfy this property and as a consequence they develop a shock, as we shall see in the following.

Square-wave initial conditions. Consider first the initial datum given by the following rectangular pulse of width $2 L$ :

$$
\begin{aligned}
& \rho(x, 0)= \begin{cases}q_{0}^{2} & |x|<L \\
0 & |x|>L,\end{cases} \\
& u(x, 0)=0,
\end{aligned}
$$

with $q_{0}>0$, corresponding in the optics framework to an NRZ pulse. The system of equations (1.5) with initial conditions (1.9) is known in the literature as the "dambreaking" problem. (In the hydrodynamic analogy, the problem describes the behavior of a mass of water which is initially confined in a uniform, spatially localized state by two dams located at $x= \pm L$ and both of which are removed at $t=0$.) The Riemann invariants for this situation are shown as the dashed lines in Figure 2. Note that the initial data for the Riemann invariants $r_{1}, r_{2}$ corresponding to the initial condition (1.9a) is not of rarefaction type. It is possible, however, to obtain initial data of rarefaction type by properly redefining the initial value of the invariants $r_{1}$ and $r_{2}$ for $|x|>L$, as shown in Figure 2 later. (This procedure is a special case of the process known as regularization; see next section.) Then the system has the following solution up to the time $t_{0}=L / q_{0}$ : For $0<x<L+2 q_{0} t$, it is

$$
\begin{aligned}
& \rho(x, t)=\min \left\{q_{0}^{2}, \frac{1}{9}\left[2 q_{0}-(x-L) / t\right]^{2}\right\}, \\
& u(x, t)=\min \left\{0,-\frac{2}{3}\left[q_{0}+(x-L) / t\right]\right\},
\end{aligned}
$$

while for $x>L+2 q_{0} t, \rho(x, t)=u(x, t)=0$, with $\rho(-x, t)=\rho(x, t)$ and $u(-x, t)=-u(x, t)$ (cf. [27]). The full solution of the NLS equation (1.1) with initial condition (1.9a), as obtained from numerical simulations (described in Section 6) is depicted in Figure 1a. (This kind of solution is usually called a fan in the context of hydrodynamics.) The speeds of the boundaries of the top $\left(\rho(x, t)=q_{0}^{2}\right)$ and bottom $(\rho(x, t)=0)$ regions are easily obtained from (1.10) (see also Figure 2b later); these two speeds are respectively $s_{2}^{-}=q_{0}$ and $s_{2}^{+}=-2 q_{0}$.

Equations (1.10) cease to be valid beyond the time $t_{0}=L / q_{0}$ when the boundaries of the top region meet at $x=0$ (i.e., the time at which the left-moving characteristic emanating from $x=L$ meets with the right-moving characteristic from $x=-L$ ). An 

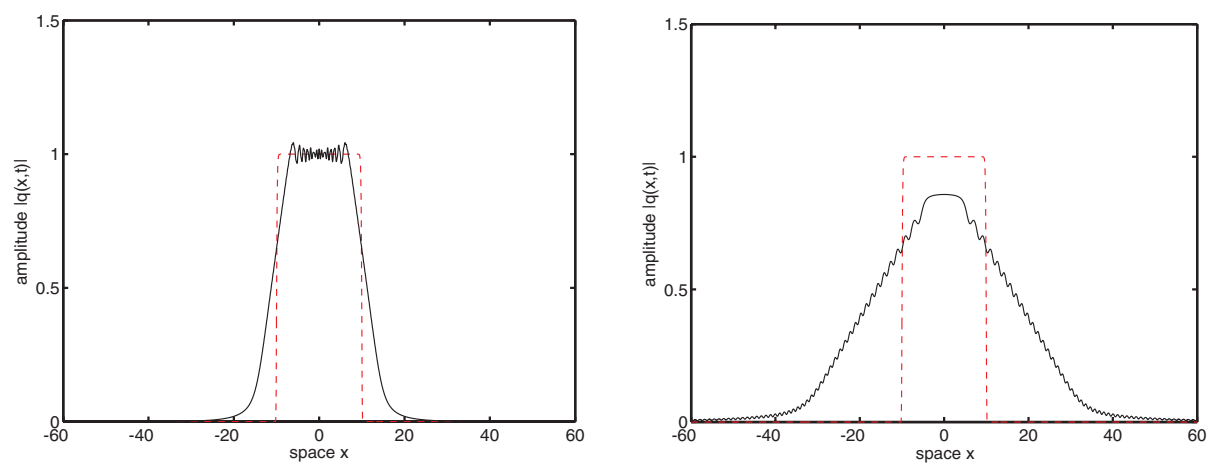

Fig. 1. Deformation of an NRZ pulse (i.e., the "dam-breaking" problem) defined by the initial conditions in (1.9a), with $q_{0}=1, L=20$, and $\epsilon^{2}=0.1$ : (a) $t^{\prime}=10$; (b) $t^{\prime}=40$, where $t^{\prime}=t / \epsilon$. The critical time here is $t_{0}=L / q_{0}=20$; hence (a) and (b) show the solution respectively before and after the time $t_{0}$. The dotted lines show the initial condition, while the solid lines show the result of numerical simulations of the NLS equation (1.1), which are performed in terms of the fast time scale $t^{\prime}$, as discussed in Section 6.

analytical expression for $q(x, t)$ when $t>L / q_{0}$, however, can be obtained using the dispersionless limit of the scattering transform for the NLS equation [33]. The corresponding behavior of the full solution of the NLS equation is shown in Figure 1b. The appearance of small oscillations in the numerical solution in Figure 1 was discussed in [16], and is the consequence of approximating the discontinuous initial data (1.9a) with a continuous initial datum in the numerical simulations (as described in Section 6). It should also be noted that some care must be taken regarding the regularization of the discontinuous initial datum (1.9a) for (1.5), since a weak solution with a discontinuity is not unique. In fact, one can construct a different solution of (1.4) with a discontinuity. In our case, however, the regularization described in Section 2 enforces the continuity of $\rho(x, t)$, thus removing the ambiguity and producing a unique solution.

Frequencyjumps and high-frequency oscillations. In terms of optical pulses, the above results imply that the initial condition in (1.9a) rapidly spreads out, as shown in Figure 1. This behavior can be partly prevented (or, alternatively, reinforced) by employing initial conditions with nontrivial phase. For example, consider the following:

$$
u(x, 0)= \begin{cases}-u_{0} & x<0 \\ u_{0} & x>0\end{cases}
$$

with $\rho(x, 0)$ still given by (1.9a). Hereafter, we will use $r_{1}^{0}(x)$ and $r_{2}^{0}(x)$ to refer to the value of the Riemann invariants (1.7) at $t=0$. If $\rho(x, 0)$ is given by $(1.9 \mathrm{a})$ and $u(x, 0)$ by $\left(1.9 \mathrm{~b}^{\prime}\right)$, for $|x|<L$ we have

$$
r_{1}^{0}(x)= \begin{cases}-u_{0}-2 q_{0} & x<0 \\ u_{0}-2 q_{0} & x>0\end{cases}
$$




$$
r_{2}^{0}(x)= \begin{cases}-u_{0}+2 q_{0} & x<0, \\ u_{0}+2 q_{0} & x>0,\end{cases}
$$

as shown by dashed lines in Figure 4. Note that the value of the invariants can be redefined for $|x|>L$, since $\rho(x, 0)=0$ there.

In terms of optical pulses, $\left(1.9 \mathrm{~b}^{\prime}\right)$ amounts to imposing a frequency jump at the center of the pulse $(x=0)$. If $u_{0}>0$, the right half of the pulse acquires a positive frequency and the left half a negative frequency. Thus, owing to the normal dispersion, the two halves of the pulse will tend to move towards each other [28]. In terms of the hydrodynamical problem, this corresponds to assigning an inward initial velocity to the mass of water, as if two pistons were acting on each side of it. (For this reason, this case is often referred to as the "piston" problem.) Note that if $u_{0}>0$, the initial data $r_{1,2}^{0}(x)$ are increasing. Thus, another consequence of the initial frequency jump is that if $u_{0}>0$, a shock develops at $x=0$, and the solution develops high-frequency oscillations, as shown in Figure 3 . (This type of shock is called collisionless, or dispersive, to distinguish it from the usual type of shock, which is dissipative; e.g., see [36].) The characteristic frequency of these oscillations is $O(1 / \epsilon)$ (i.e., one period of the oscillation shown in Figure 3 is of order $\epsilon$ ). If $u_{0}<0$ instead, the two halves of the pulse will move away from each other, and no shocks develop in this case. (In hydrodynamics, solutions such as this one are called of rarefaction type.) When the solution develops a shock, the hyperbolic system (1.5) ceases to be valid, and the solution of the NLS equation in the weak dispersion limit must be obtained by properly regularizing the hyperbolic system, as we briefly discuss next.

\section{Regularization and the NLS-Whitham equations}

The semiclassical limit of the NLS equation has been studied extensively in the last fifteen years; e.g., see [15], [24], [25], [26], [27], [29], [31], [33] for different approaches, such as the connection with the integrable character and the multi-phase solutions of the NLS equation and with Whitham's averaging method. A self-contained description of the regularization process can be found in [27], together with a detailed treatment of the situation in which the initial condition contains only one frequency jump. Here we will limit ourselves to presenting a brief general overview and recalling some results that are relevant for the remainder of this work. Some additional details (which are necessary to perform the calculations described in Sections 3 and 4) are contained in Appendices A.2 and A.3.

As we will see throughout this work, for certain kinds of initial conditions the solution of the NLS equation (1.1) with small dispersion develops high-frequency oscillations. When this happens, the approximate hyperbolic system (1.5) is inadequate to describe the dynamics of the solution, because strong dispersive effects appear due to the presence of high-frequency oscillations. An effective way to describe the behavior of the solution of the NLS equation in these situations is obtained by taking an average over these high-frequency oscillations via the Whitham technique, which consists in locally approximating the solution by finite-genus solutions of the NLS equation, then describing the global behavior as a slow modulation of this local periodic or quasi-periodic structure. 
The evolution of these modulations is then governed by the Whitham equations, which are obtained by averaging the conservation laws of the NLS equation over one period of the fast oscillations, and which express the local average of the quasi-periodic solutions with respect to these fast oscillations (cf. [14], [35], [36] for the Korteweg-de Vries equation).

Recall that the hyperbolic system (1.6) is a dispersionless limit of the NLS equation. The presence of high-frequency oscillations in the solution of the NLS equation corresponds to the formation of a shock singularity in (1.6) generating strong dispersion, and the Whitham averaging technique then provides an appropriate dispersive regularization of this singularity. This regularization turns out to consist of enlarging the system (1.6) to include more than two Riemann invariants (as given by (2.3) below) in such a way that the initial data for the enlarged system becomes of rarefaction type, which in turn implies that the system possesses a global solution for all values of time. The solution of the original problem is then described in terms of the solution of an NLS-Whitham equation of finite genus. The dispersionless equation (1.6) corresponds to the genus-zero NLS-Whitham equation. The value of the genus is determined by the specifics of the initial condition considered, since these determine the number of Riemann invariants that are needed to regularize the hyperbolic system.

Let us briefly review some features of the regularization and introduce the NLSWhitham equations (see Appendices A.2 and A.3 and [15], [24], [27], [29] for more details). It is well-known that the NLS equation is integrable via the inverse scattering transform. The scattering problem associated with the defocusing NLS equation (1.1) is given by the eigenvalue problem [2], [37] $\mathcal{L} \mathbf{v}=z \mathbf{v}$, where $\mathbf{v}$ is a two-component vector, $z \in \mathbb{C}$ is the spectral parameter of the scattering problem, and the Lax operator $\mathcal{L}$ is defined by

$$
\mathcal{L} \mathbf{v}=\left(\begin{array}{cc}
-i \epsilon \partial_{x} & i q \\
-i q^{*} & i \epsilon \partial_{x}
\end{array}\right) \mathbf{v}
$$

A genus- $g$ solution of the NLS equation is associated with a genus- $g$ hyperelliptic Riemann surface $R: w^{2}=\mu_{g}(z)$, with

$$
\mu_{g}(z)=\prod_{k=1}^{2 g+2}\left(z-r_{k}\right)
$$

The solution of the NLS equation (1.1) corresponding to (2.2) is described in terms of a Baker-Akhiezer function constructed from Riemann theta functions with $g$ phases (see [6], [18] for details). The branch points $r_{1}, \ldots, r_{2 g+2}$ of $R$ determine the spectrum of $\mathcal{L}$. Since the Lax operator $\mathcal{L}$ is self-adjoint, these branch points are all real, and we label them so that $r_{1}<r_{2}<\cdots<r_{2 g+2}$. The spectrum of $\mathcal{L}$ is then given by $\left(-\infty, r_{1}\right] \cup\left[r_{2}, r_{3}\right] \cup \cdots \cup\left[r_{2 g}, r_{2 g+1}\right] \cup\left[r_{2 g+2}, \infty\right)$; the bounded intervals of the spectrum (that is, $\left[r_{2 k}, r_{2 k+1}\right]$ for $k=1, \ldots, g$ ) are called the gaps.

For an exact genus- $g$ solution of the NLS equation, the branch points $r_{1}, \ldots, r_{2 g+2}$ are obviously constant, independent of space and time, owing to the isospectrality of the inverse scattering transform of the NLS equation. The solution then gives a quasiperiodic solution with $g$ phases of the NLS equation, and with a small dispersion of order $\epsilon^{2}$ implies that each period of the phase is of order $\epsilon$ (i.e., high-frequency oscillations of order $1 / \epsilon)$. 
Suppose now that the $g$-phase solution is slowly modulated in space. Then the spectral parameters $r_{k}$ are expected to shift slightly, (Moreover, additional openings in the spectrum, or gaps, can also form in general; see for example Sections 3 and 4.) We can treat the modulation problem using a singular perturbation method as follows: If we consider modulations on the scale of $(x, t)$ in the NLS equation (1.1), the modulations are of order one, and the oscillations are of order $1 / \epsilon$. This implies that one can treat the modulation problem as a small perturbation of the $g$-phase solution, which is the key of the Whitham averaging method. This method is based on an adiabatic assumption that the leading-order solution of the NLS equation (i.e., the $g$-phase solution) is preserved under the modulation, but with slowly changing parameters. That is, the shifted parameters are constant as the eigenvalues of the Lax operator $\mathcal{L}$. Employing a standard averaging perturbation method, one then introduces the fast and slow time and space scales respectively as $\left(x^{\prime}=x / \epsilon, t^{\prime}=t / \epsilon\right)$ and $(x, t)$. The first-order correction to the $g$-phase solution in the perturbation method compensates the motion of the parameters so that the parameters appear to be constant over regions of order one in $(x, t)$, but at the same time acquire small constant shifts due to the modulations in general. The corresponding equations for the spectral parameters with respect to the slow scales $(x, t)$ are called the NLS-Whitham equations, or simply the Whitham equations, and are obtained by averaging the conservation laws of the NLS equation with respect to the fast oscillations at order $\epsilon$ (see Appendix A.3 for more details).

It was shown in [15], [29] that the genus- $g$ Whitham equations for the NLS equation can be written in the Riemann invariants form, in which the spectral parameters $r_{k}$ give the Riemann invariants,

$$
\frac{\partial r_{k}}{\partial t}=s_{k}\left(r_{1}, \ldots, r_{2 g+2}\right) \frac{\partial r_{k}}{\partial x}
$$

for $k=1, \ldots, 2 g+2$. It then follows that for each $\epsilon \neq 0$ the solution of the NLSWhitham equations describes the slow modulation of finite-genus solutions of the NLS equation. The following was also shown (cf. Lemma 4.1 in [27]), and is the most important property of the NLS-Whitham equations:

Proposition 2.1. The characteristic velocities $s_{k}$ possess a double sorting property, i.e., $\forall k, l=1, \ldots, 2 g+2$. They satisfy the two conditions,

$$
\begin{aligned}
\frac{\partial s_{k}}{\partial r_{k}} & >0, \\
r_{k} & <r_{l} \Rightarrow s_{k}<s_{l},
\end{aligned}
$$

Then, as a consequence of (2.1), we have (Corollary 4.2 in [27]):

Corollary 2.2. If the initial values of the $r_{k}$ are each nonincreasing, and if they satisfy the separability condition

$$
\max _{x \in \mathbb{R}} r_{k}^{0}(x)<\min _{x \in \mathbb{R}} r_{k+1}^{0}(x),
$$

$\forall k=1, \ldots, 2 g+1$ at $t=0$, the initial data is of rarefaction type, and therefore the hyperbolic system of equations (2.3) has a global solution for $t>0$, i.e., it is regular. 
In general, the initial conditions for the two Riemann invariants obtained from the dispersionless system (1.4) do not satisfy monotonicity and the separability condition (2.5). In other words, (1.6) (that is, the system (2.3) with $g=0$ ) in general do not have a global solution. The regularization process then consists of enlarging the set of Riemann invariants so that the resulting NLS-Whitham equations have a global solution. This is done by representing the initial data for the NLS eq. (1.1) in such a way that all the Riemann invariants $r_{1}, \ldots, r_{2 g+2}$ are monotonically decreasing functions of $x$ at $t=0$. Note that it is always possible to do so for piecewise-constant initial data, since in this case there is some ambiguity in how the spectrum of the Lax operator is represented in terms of the Riemann invariants. This ambiguity can then be exploited to redefine the initial datum for the Whitham eqs. (2.3) by adding degenerate gaps (i.e., degenerate openings between the invariants; e.g., see Figures 4 and 6). All of the solutions discussed in Sections 3, 4, and 5 fall within the framework of piecewise-constant initial conditions. In this case the data at $t=0$ are always genus- 0 , but they can be described in terms of highly degenerate higher-genus data, namely, higher-genus data with degenerate gaps.

It should be noted that the adiabatic approximation (the use of the Whitham equations for the slow evolution) implies that the local genus of the solution is preserved. A separate issue is how the genus of the solution changes from one region to the next. This is a bifurcation problem through a critical point, and can be approached by regularization, i.e., by trying to patch two Whitham systems with different genus at this point. Our regularization acts as a "globalization," in the sense that the Whitham equations with a proper (in general larger) genus now describes a global behavior beyond the perturbation range. For fixed $x$, a regular scheme must solve a connection problem in $t$ because of the change of genus, and for fixed $t$ one also needs to solve a connection problem to match regions with different genus.

It is also important to realize that for piecewise-constant initial data there is more than one way to regularize the initial datum (e.g., see Figure 2). The minimum number of Riemann invariants that are necessary so that the system becomes regular is related to the genus of the solution of the NLS-Whitham equations. That is, $2 g+2$ invariants correspond to a genus- $g$ solution of (2.3). The local genus of the solution of the NLS equation is roughly speaking the number of distinct frequencies that are locally present in the solution over regions of order one in $(x, t)$. Since the Riemann invariants are the branch points of the spectrum of the finite-genus solution of the NLS equation that locally approximates the full solution, it is then clear that the local genus is equal to the number of gaps (i.e., bounded intervals of the spectrum of the Lax operator $\mathcal{L}$ ) corresponding to the local value of the Riemann invariants (e.g., see Figures 8 and 9). The opening or closing of one of the gaps for some values of $(x, t)$ corresponds to a local change of genus in the solution of the NLS equation. Note however that all finite-genus solutions of the NLS equation with nonzero genus become singular in the limit $\epsilon \rightarrow 0^{+}$. In this sense, the solution of the Whitham equations represents a weak limit, since when $g \neq 0$ the solutions of the NLS equation only converge in an average sense (i.e., weak convergence).

Finally, with regards to Figure 2, we should note that genus-1 data is necessary in order to preserve the value of $u(x, 0)$. With step initial data, however, no gap opens during propagation, which means that the data is degenerate, resulting in a genus-0 solution. The situation would be different in the case of nonstep initial data (e.g., if the transition 


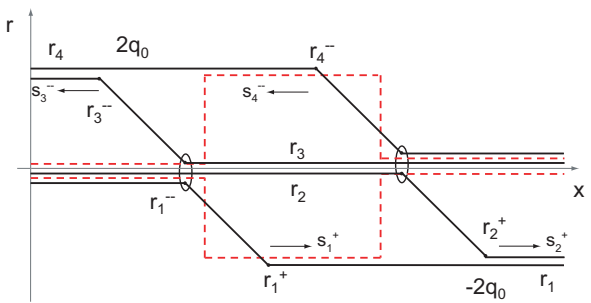

(a)

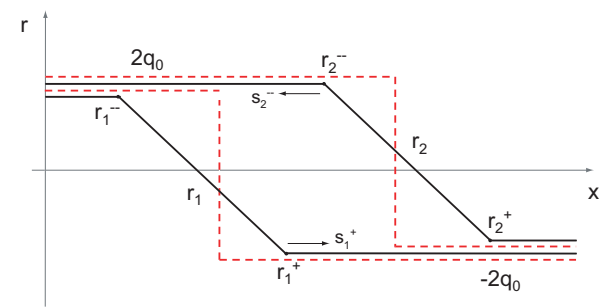

(b)

Fig. 2. Evolution of the Riemann invariants in two equivalent cases. Dashed lines: the Riemann invariants at $t=0$; solid lines: the invariants at $t \neq 0$. (a) corresponds to the square-wave initial datum in (1.9a) and Figure 1, regularized by genus-1 data; (b) shows an equivalent diagram of the left one, and it is given by genus-0 data. Hereafter, the subscripts "-" and "+" refer to the value of the invariants respectively to the left and to the right of their initial discontinuity. The ellipses in (a) indicate "locking" points, i.e., points $x_{*}$ for which $r_{1}\left(x_{*}, t\right)=r_{2}\left(x_{*}, t\right)=r_{3}\left(x_{*}, t\right)$ (on the left) and $r_{2}\left(x_{*}, t\right)=r_{3}\left(x_{*}, t\right)=r_{4}\left(x_{*}, t\right)$ (on the right) for all $t$. This implies that the regularization at those points is trivial. Finally, note that the initial conditions $r_{1,2}^{0}(x)$ in (b) have been redefined whenever $\rho(x, 0)=0$ (i.e., $u(x)$ itself is not defined in the NLS-Whitham equation when $\rho(x)=0$, see (A.9)).

from $\rho(x, 0)=0$ to $\rho(x, 0)=q_{0}^{2}$ were continuous); in that case, oscillations would appear, as described in [16].

\section{Single-Jump Initial Conditions}

We now briefly summarize some results from [27] relative to a single-jump initial datum, since they provide the basis for the framework that will be used to analyze the more complicated scenarios discussed in the remainder of this work. We will consider the constant-amplitude wave given by the (single-jump) initial condition in (1.9a) and (1.9 $\mathrm{b}^{\prime}$ ), where we take $L \rightarrow \infty$. The value of the original Riemann invariants in the genus-0 system (1.6) at $t=0$ is again given by (1.11), which are now valid $\forall x \in \mathbb{R}$. Four different situations arise depending on the size of the frequency jump $2 u_{0}$, as shown in Figure 3:

(i) $u_{0}>2 q_{0}>0$ (Lemma 4.3 and Theorem 4.4 in [27]).

Since $u_{0}>0$, the original Riemann invariants $r_{1,2}^{0}(x)$ are increasing functions of $x$, and therefore the genus-0 system (1.6) does not have a global solution. In this case the problem is regularized by considering the genus-1 NLS-Whitham equations. (That is, four invariants are necessary so that the resulting system has a global solution.) The Riemann invariants are related to the solution at $t=0$ as follows:

$$
\begin{aligned}
& r_{1}=-u_{0}-2 q_{0}, \quad \forall x, \quad r_{3}=u_{0} \pm 2 q_{0}, \quad \text { for } x \lessgtr 0, \\
& r_{2}=-u_{0} \pm 2 q_{0}, \quad \text { for } x \lessgtr 0, \quad r_{4}=u_{0}+2 q_{0}, \quad \forall x,
\end{aligned}
$$




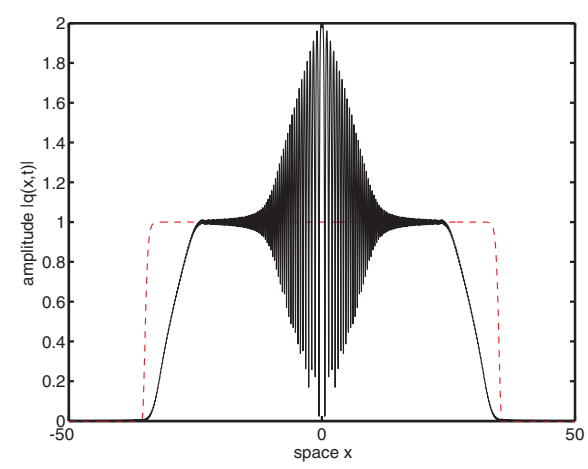

(a)

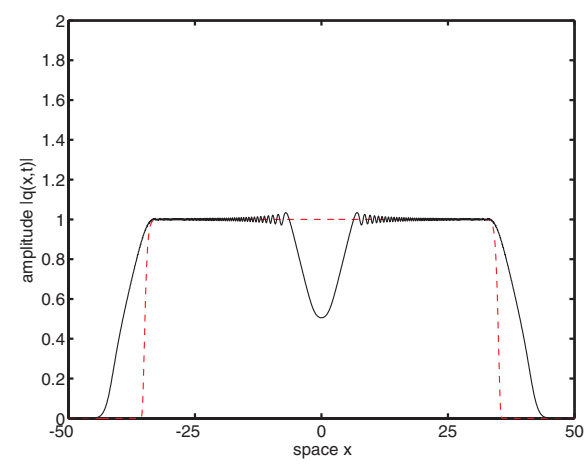

(c)

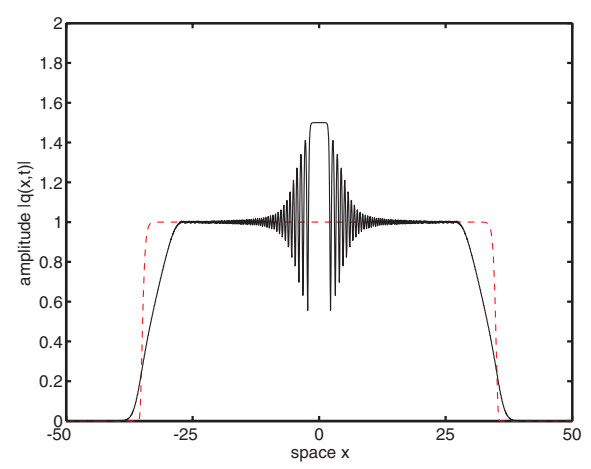

(b)

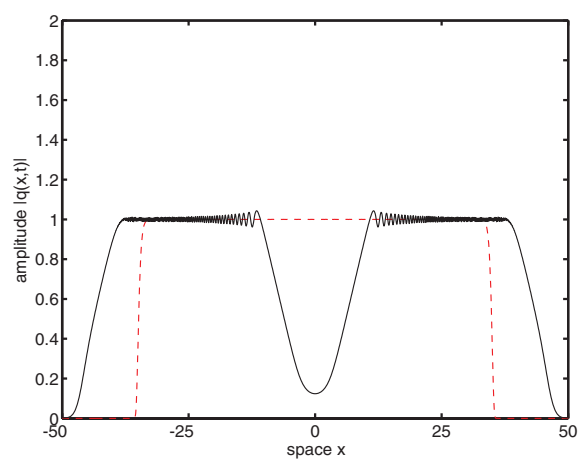

(d)

Fig. 3. Deformation of an NRZ pulse in the presence of an initial frequency jump: (a) $u_{0}=2.01$; (b) $u_{0}=1$; (c) $u_{0}=-1$; (d) $u_{0}=-2.5$. The dotted line shows the initial condition; the solid line shows the result of numerical simulations of the NLS equation, performed as discussed in Section 6. In all four cases, it is $q_{0}=1, L=35$, and $\epsilon^{2}=0.1$, and the solution is shown at $t^{\prime}=20$, where $t^{\prime}=t / \epsilon$.

with the upper/lower signs corresponding to the upper/lower inequality for $x$, respectively. The qualitative evolution of these Riemann invariants is shown in Figure 4a. The solution develops a region of genus-1 high-frequency oscillations in the central portion of the pulse, surrounded by a genus- 0 region, as illustrated in Figure 3a and Figure 5a. As shown in Figure 3a, the genus-1 portion of the solution describes slow modulations of high-frequency oscillations, as would be the case in a wave packet. The genus- 1 portion of the solution is located in the region $|x|<s_{3}^{-} t$. The characteristic velocities are $s_{3}^{-}=-s_{2}^{+}, s_{3}^{+}=-s_{2}^{-}$, with $s_{3}^{-}>s_{3}^{+}>0$, and

$$
\begin{aligned}
& s_{3}^{-}=\left(u_{0}+q_{0}\right)\left[1+u_{0} q_{0} /\left(u_{0}+q_{0}\right)^{2}\right], \\
& s_{3}^{+}=u_{0}\left[1-3 a(1-a) K_{1}-2 a^{2} K_{2}\right] /\left(1-a K_{1}\right)
\end{aligned}
$$




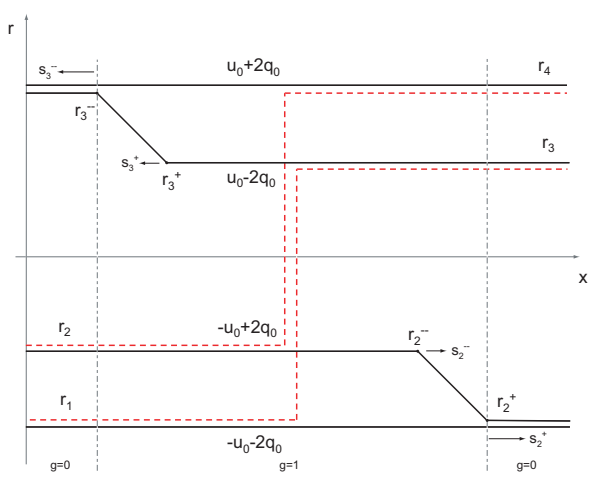

(a)

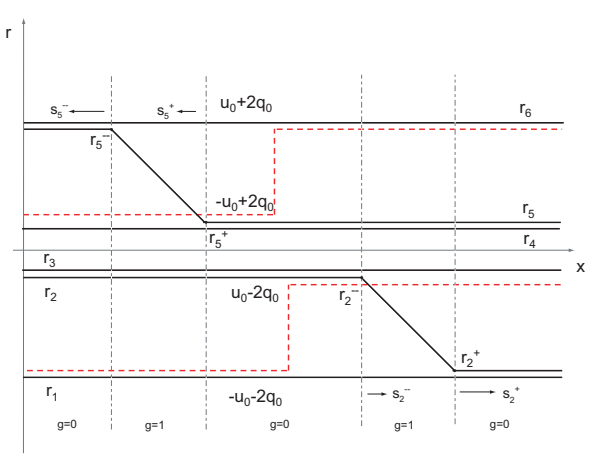

(b)

Fig. 4. Qualitative diagrams illustrating the evolution of the Riemann invariants: (a) $u_{0}>2 q_{0}$, with a single, expanding genus- 1 region; (b) $0<u_{0}<2 q_{0}$, with an expanding genus- 0 region surrounded by expanding genus- 1 regions on either side. Dashed lines: the original invariants $r_{1,2}^{0}(x)$ at $t=0$; solid lines: the regularized invariants at $t \neq 0$; dot-dashed vertical lines: boundaries between regions of different genus. Note that the connecting segments between $r_{2}^{ \pm}$in Figure $4 \mathrm{a}, \mathrm{b}$, $r_{3}^{ \pm}$in Figure $4 \mathrm{a}$, and $r_{5}^{ \pm}$in Figure $4 \mathrm{~b}$ are actually curved, and are represented here only by straight lines for simplicity (e.g., see [8]).

(cf. (4.16) and (4.17) in [27]), where $a=2 q_{0} / u_{0}<1$, and

$$
1-K_{n}=\frac{\int_{0}^{\pi / 2} \frac{\sin ^{2 n+2} \theta}{\sqrt{\left(1+a \sin ^{2} \theta\right)\left(1+a \cos ^{2} \theta\right)}} d \theta}{\int_{0}^{\pi / 2} \frac{\sin ^{2} \theta}{\sqrt{\left(1+a^{2} \sin ^{2} \theta\right)\left(1+a^{2} \cos ^{2} \theta\right)}} d \theta} .
$$

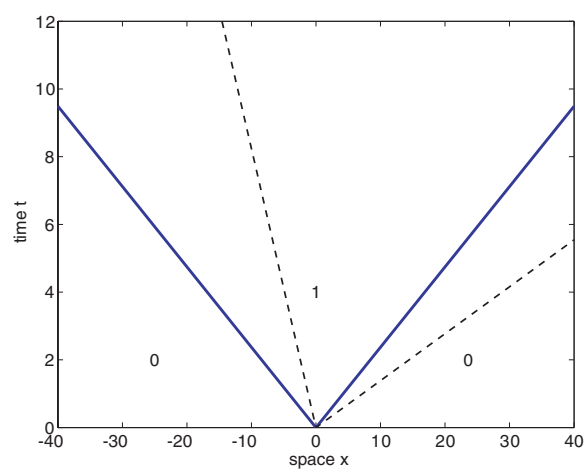

(a)

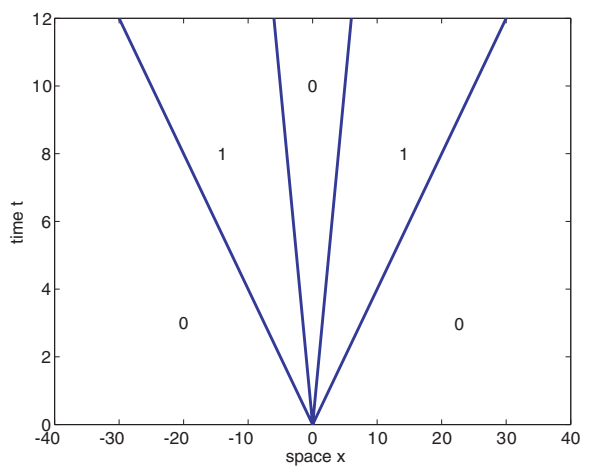

(b)

Fig. 5. The boundaries between regions of genus- 0 and genus- 1 in the $(x, t)$-plane corresponding to the cases shown in Figure 4: (a) $u_{0}=5 / 2$, corresponding to case (i), and Figures $3 \mathrm{a}$ and 4a; (b) $u_{0}=1$, corresponding to case (ii) and Figures $3 \mathrm{~b}$ and $4 \mathrm{~b}$. Dashed lines: the same boundaries after adding a constant frequency offset $u_{\text {avg }}=3$ to both sides of the jump, as discussed in Section 4. 
Hereafter, the superscripts "-" and "+" refer to the value of the Riemann invariants respectively to the left and to the right of the discontinuities at $t=0$ (cf. Figure 4). Note that, owing to (2.3), positive values of $s$ correspond to left-moving Riemann invariants, and that for regularized, nonincreasing invariants $r^{-}(x) \geq r^{+}(x)$ $\forall x \in \mathbb{R}$.

(ii) $0<u_{0}<2 q_{0}$ (Lemma 4.5 and Theorem 4.6 in [27]).

Again, since $u_{0}>0$, the genus- 0 Whitham equations do not have a global solution. This case is regularized by the genus-2 NLS-Whitham equations. The six Riemann invariants are related to the solution at $t=0$ as follows:

$$
\begin{array}{ll}
r_{1}=-u_{0}-2 q_{0}, \quad \forall x, & r_{4}=-u_{0}+2 q_{0}, \quad \forall x, \\
r_{2}= \pm u_{0}-2 q_{0}, \quad \text { for } x \lessgtr 0, & r_{5}= \pm u_{0}+2 q_{0}, \quad \text { for } x \lessgtr 0, \\
r_{3}=u_{0}-2 q_{0}, \quad \forall x, & r_{6}=u_{0}+2 q_{0}, \quad \forall x .
\end{array}
$$

The qualitative evolution of these Riemann invariants is shown in Figure 4b. Even though $g=2$ is necessary to regularize the initial data, no genus- 2 region appears. The solution develops a genus-0 flat region (a degenerate genus- 2 region) in the central portion of the pulse, surrounded by genus-1 high-frequency oscillations, as illustrated in Figure 3b. The genus- 0 and genus- 1 portions of the solution are respectively located in the regions $|x|<s_{5}^{+} t$ and $s_{5}^{+} t<|x|<s_{5}^{-} t$, with $s_{5}^{+}=-s_{2}^{-}$, $s_{5}^{-}=-s_{2}^{+}$, and where $s_{5}^{-}>s_{5}^{+}>0$. The characteristic velocities are (eqs. (4.28) in [27])

$$
s_{5}^{+}=q_{0}-\frac{1}{2} u_{0}, \quad s_{5}^{-}=\left(u_{0}+q_{0}\right)\left[1+u_{0} q_{0} /\left(u_{0}+q_{0}\right)^{2}\right]
$$

Furthermore, in the genus- 0 region, the solution takes the value $\rho=\frac{1}{16}\left(r_{2}^{0}-r_{1}^{0}\right)^{2}$, i.e.,

$$
\rho=q_{0}^{2}\left[1+u_{0} / 2 q_{0}\right]^{2} .
$$

Note that the amplitude of the genus-0 (nonoscillatory) region at the center increases with increasing modulation strength $u_{0}$. At the same time, however, the dependence of $s_{5}^{+}$on $u_{0}$ implies that the width of the genus- 0 region decreases with increasing modulation strength, and the region ceases to exist when $u_{0}>2 q_{0}$. The maximum possible amplitude that can be obtained in this way is thus $\rho_{\max }=4 q_{0}^{2}$, obtained for $u_{0}=2 q_{0}$.

This case, $0<u_{0}<2 q_{0}$, is the most interesting case for applications because, unlike the high-frequency oscillations in the genus-1 portion, the high-amplitude genus-0 region can survive an appropriate filtering and produce short, high-intensity optical pulses. As mentioned above, however, the maximum amplitude that can be obtained with this arrangement is limited, i.e., $q=\left(1+u_{0} / q_{0}\right) q_{0}<2 q_{0}$. In the next section we will see how this limit on the maximum pulse amplitude can be overcome by employing more than one frequency jump.

(iii) $-2 q_{0}<u_{0}<0$ (Theorem 4.7 in [27]).

Since $u_{0}<0$, the Riemann invariants $r_{1,2}^{0}(x)$ are nonincreasing functions of $x$, and they satisfy the separability condition (2.5). Therefore the genus-0 NLS-Whitham equations (1.5) with the Riemann invariants defined as in (1.7) has a global solution, 
and no regularization is necessary. The solution develops a depression zone in the center, as illustrated in Figure 3c. More precisely (eqs. (4.31) and (4.33) in [27]),

$$
\rho(x, t)= \begin{cases}q_{0}^{2}\left[1+u_{0} /\left(2 q_{0}\right)\right]^{2}, & |x|<s_{2}^{+} t, \\ {\left[x+\left(u_{0}+2 q_{0}\right) t\right]^{2} / 9 t^{2},} & s_{2}^{+} t<|x|<s_{2}^{-} t, \\ q_{0}^{2}, & |x|>s_{2}^{-} t,\end{cases}
$$

where

$$
s_{2}^{+}=\frac{1}{2}\left(u_{0}+2 q_{0}\right), \quad s_{2}^{-}=q_{0}-u_{0} .
$$

(iv) $u_{0}<-2 q_{0}$ (Lemma 4.8 and Theorem 4.9 in [27]).

Even though the invariants are nonincreasing, they do not satisfy the separability condition (2.5). This case is regularized by the genus-1 NLS-Whitham equations (1.5). The Riemann invariants at $t=0$ are defined by

$$
\begin{aligned}
& r_{1}=u_{0} \pm 2 q_{0}, \quad \text { for } x \lessgtr 0, \quad r_{3}=-u_{0}-2 q_{0}, \quad \forall x \\
& r_{2}=u_{0}+2 q_{0}, \quad \forall x, \quad r_{4}=-u_{0} \pm 2 q_{0}, \quad \text { for } x \lessgtr 0 \text {, }
\end{aligned}
$$

The corresponding solution is shown in Figure 3d. Even though $g=1$ is necessary to regularize the initial data, no gap opens among the invariants, and therefore this case results in a degenerate, genus- 0 solution. In other words, no genus- 1 regions develop. (The high-frequency oscillations visible in Figure $3 \mathrm{~d}$ disappear in the limit $\epsilon \rightarrow 0^{+}$in the case of step initial data.) The solution is similar to the one in case (iii), except that $q(x, t)$ tends to zero at $x=0$. (The nonzero value of $q(0, t)$ in Figure $3 \mathrm{~d}$ is due to the finiteness of $\epsilon$ in the numerical simulations.) More precisely, $\rho(x, t)=q_{0}^{2}$ for $|x|>s_{4}^{-} t$ and $\rho(x, t)=0$ in the limit $\epsilon \rightarrow 0^{+}$in the region $|x|<s_{4}^{+} t$, where (eqs. (4.36) in [27])

$$
s_{4}^{+}=-u_{0}-2 q_{0}, \quad s_{4}^{-}=-u_{0}+q_{0} .
$$

The calculation of the characteristic speeds for the Riemann invariants is based on the formulation of the NLS-Whitham equations. We refer the reader to Appendices A.3 and A.4 and to [7], [8], [27] for further details. Note that the outer boundaries of the genus- 1 region are given by $x= \pm s_{\text {outer }} t$, where $s_{\text {outer }}$ is the same in case (i) and case (ii): $s_{\text {outer }}=\left(u_{0}+q_{0}\right)\left[1+u_{0} q_{0} /\left(u_{0}+q_{0}\right)^{2}\right]$ (cf. $s_{3}^{-}$in (3.1) and $s_{5}^{-}$in (3.2)). Note also that the location of the boundaries between regions of genus- 0 and genus- 1 in the numerical simulations shown in Figures 3a-d agrees very well with the analytical results just presented, even though the value of $\epsilon$ used is not very small.

Case (ii) is the most interesting for applications because of the high-amplitude genus0 region. Also, in cases (iii) and (iv) (i.e., when $u_{0}<0$ ), the solution is only of genus- 0 , but lower amplitude. For this reason we will mainly focus our attention on the case of positive frequency jumps. Hereafter, we refer to case (ii) as a "subcritical" frequency jump and to case (i) as a "supercritical" frequency jump. More precisely:

Definition 3.1. We say that a single frequency jump located at $x=x_{0}$ is supercritical if $r_{1}^{0}\left(x_{0}^{+}\right)>r_{2}^{0}\left(x_{0}^{-}\right)$, subcritical if $r_{1}^{0}\left(x_{0}^{+}\right)<r_{2}^{0}\left(x_{0}^{-}\right)$, where as usual $r_{k}^{0}\left(x^{ \pm}\right)=$ $\lim _{\Delta x \rightarrow 0^{ \pm}} r_{k}^{0}(x+\Delta x)$. 
In the following sections we generalize the above solutions and discuss the behavior of solutions of the finite-genus NLS-Whitham equations in the presence of an arbitrary number of jumps in the initial data.

\section{Behavior of Finite-Genus Solutions: Two Frequency Jumps}

More complicated situations than those described in the previous section arise when the initial conditions contain more than one frequency jump. The main purpose of this and the following section is to study the interaction among finite-genus solutions of the NLS-Whitham equations generated by those frequency jumps. First of all, however, let us briefly discuss the effect of adding a nonzero average frequency across the jump. The Galilean invariance of the NLS equation implies that it is possible to redefine the local frequency $u(x, 0)$ up to an arbitrary additive constant. The effect of such a constant, representing a global frequency translation, is just a change in the overall group velocity of the solution. More precisely, if $q_{o}(x, t)$ is a solution of (1.1), so is

$$
q_{c}(x, t)=\exp \left[i\left(c x-c^{2} t / 2\right) / \epsilon\right] q_{o}(x+c t, t)
$$

for any constant $c$. Corresponding to (4.1) there exists a Galilean symmetry of the NLSWhitham equations (2.3) for the Riemann invariants: namely, if $r_{j}(x, t)$ is a solution of the Whitham equations (2.3), then so is the translated solution $r_{j}(x+c t, t)+c$. Note from (4.1) that a positive frequency $c / \epsilon$ corresponds to a negative shift $-c$ in the group velocity $d x / d t$. Also, as an effect of the Galilean transformation (4.1), the rescaled frequency of the transformed solution is shifted by $c$, i.e., $u_{c}(x, t)=\epsilon \partial_{x}\left[\arg q_{c}(x, t)\right]=$ $c+\epsilon \partial_{x}\left[\arg q_{o}(x+c t, t)\right]$. In terms of the decomposition of the $(x, t)$-plane into regions of different genus, we have the following:

Lemma 4.1. The boundaries between genus-0 and genus-1 regions for a single frequency jump upon adding a nonzero average frequency $u_{\text {avg }}$ to the initial condition are given by $x=s_{ \pm} t$, where $s_{ \pm}= \pm s_{\mathrm{old}}+u_{\mathrm{avg}}$, and where $s_{\mathrm{old}}$ is still given by (3.1), (3.2), (3.4), and (3.5) in cases (i)-(iv), respectively.

Thus, adding a nonzero frequency offset to both sides of a single frequency jump has the effect of tilting the corresponding boundaries between regions of different genus, as shown in Figure 5a. If only one jump is present, it is obviously possible to choose the average frequency so that both of the boundaries of the outermost genus- 0 region move in the same direction. In the presence of several frequency jumps, however, it is not always possible to do so, as will be discussed later. Because of the Galilean invariance, we will sometimes describe the initial condition for $u(x, 0)$ in terms of the frequency jumps, defined as $C_{j}=u\left(X_{j}^{+}, 0\right)-u\left(X_{j}^{-}, 0\right)$ for all $j=1, \ldots, N$, where $N$ is the total number of jumps and $X_{1}, \ldots, X_{N}$ are the jump locations.

We now turn to initial conditions with two frequency jumps. We will consider constantamplitude initial conditions with $|q(x, 0)|=q_{0} \forall x \in \mathbb{R}$. For simplicity we will take symmetric jumps of size $C_{1}=C_{2}=: 2 u_{0}$ located at $X_{2}=-X_{1}=\Delta X / 2>0$, so that 
$2 u_{0}$ is the size of each frequency jump, as in the single-jump case. That is, let

$$
u(x, 0)= \begin{cases}-2 u_{0}, & x<-\Delta X / 2, \\ 0, & |x|<\Delta X / 2, \\ 2 u_{0}, & x>\Delta X / 2\end{cases}
$$

again with $|q(x, 0)|=q_{0}>0 \forall x \in \mathbb{R}$. In the absence of either one of the two jumps, the solution would behave according to the theory described in Section 3, with the only exception being that the average frequency across the jump is now nonzero. More precisely, since the average frequency at $x= \pm \Delta X / 2$ is $\pm u_{0}$, the solutions described in the previous section would move with velocity $\mp u_{0}$. In other words, the central portion of the solutions described in Section 3 will tend to move towards each other if $u_{0}>0$ and away from each other if $u_{0}<0$. As we will see, this description provides an accurate picture of the overall solution at sufficiently small propagation times. After this initial stage, however, significant interaction effects appear.

In analogy with the calculations described in Section 3, let us now proceed to analyze the solution by looking at the Riemann invariants. The initial values $r_{1,2}^{0}(x)$ of the nonregularized Riemann invariants for the genus-0 NLS-Whitham equations (1.6) are

$$
\begin{aligned}
& r_{1}^{0}(x)= \begin{cases}-2 q_{0}-2 u_{0}, & x<-\Delta X / 2, \\
-2 q_{0}, & |x|<\Delta X / 2, \\
-2 q_{0},+2 u_{0}, & x>\Delta X / 2,\end{cases} \\
& r_{2}^{0}(x)= \begin{cases}2 q_{0}-2 u_{0}, & x<-\Delta X / 2, \\
2 q_{0}, & |x|<\Delta X / 2, \\
2 q_{0}+2 u_{0}, & x>\Delta X / 2 .\end{cases}
\end{aligned}
$$

These invariants are shown as dashed lines in Figure 6. Since expressing the values of all the regularized Riemann invariants at $t=0$ in terms of the initial datum would be rather tedious, and since all the values can be easily inferred from Figure 6, we omit the formulae for brevity.

Based on Proposition 2.1 and Corollary 2.2, the following four scenarios arise, depending on the size of the frequency jumps:

(i) $u_{0}>2 q_{0}>0$ :

This case is regularized by the genus- 2 NLS-Whitham equations (i.e., with six invariants), as illustrated in Figure 6a, where the (two) original and (six) regularized Riemann invariants are shown respectively by dashed and solid lines.

The regions of genus- 0,1 , and 2 for the solution of the NLS equation are shown in the bifurcation diagram in Figure 7a. Both jumps are supercritical individually, so two genus-1 regions open up, one at the location of each frequency jump. These two regions interact and they create a genus- 2 region at the center of the solution. The genus- 2 region then expands forever (cf. Propositions 4.4 and 4.7). In terms of the numerical simulations described in the next section, this case corresponds to Figure 11a. 


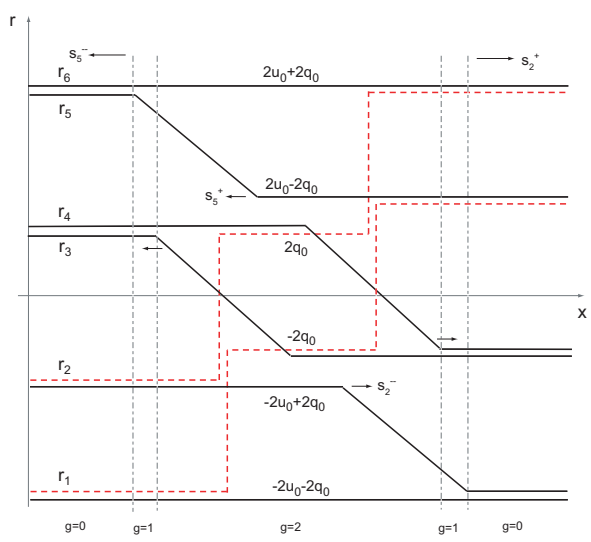

(a)

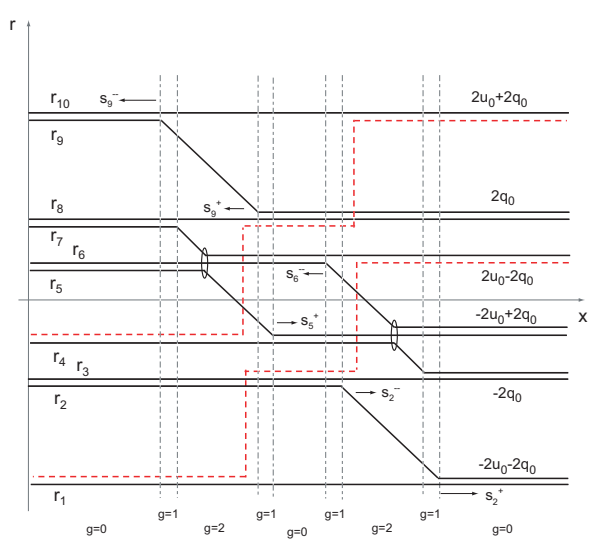

(c)

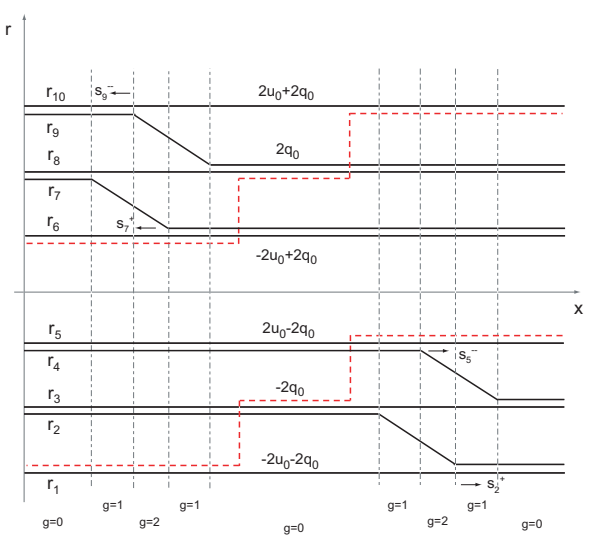

(b)

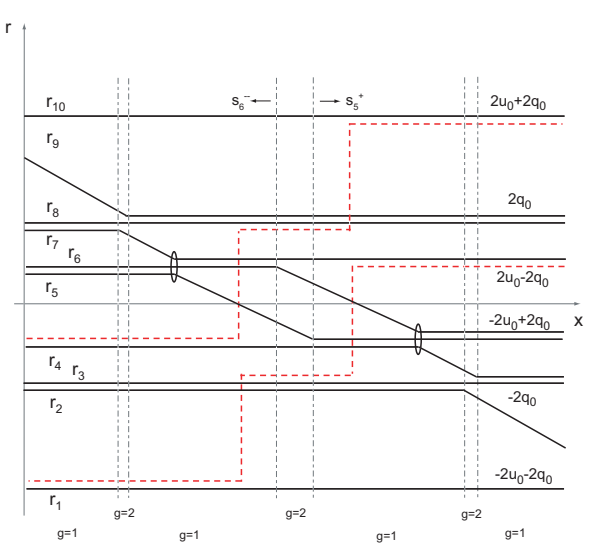

(d)

Fig. 6. Qualitative diagrams illustrating the evolution of the Riemann invariants: (a) $u_{0}>2 q_{0}$, corresponding to case (i) and showing expanding genus-1 and genus-2 regions; (b) $0<u_{0}<q_{0}$, corresponding to case (iii) and showing an expanding genus-0 central region; (c) $q_{0}<u_{0}<2 q_{0}$, corresponding to the intermediate case (ii), and showing the invariants at small time $t$ and a temporary genus- 0 region in the center; (d) the same intermediate case (i.e., $q_{0}<u_{0}<2 q_{0}$ as in (c)), but now showing the solution at large time $t$ and an expanding genus- 2 region in the center. Dashed lines: the original invariants $r_{1,2}^{0}(x)$ at $t=0$; solid lines: the regularized invariants at $t \neq 0$; dot-dashed vertical lines: boundaries between regions of different genus. As in Section 2, the ellipses in (c) and (d) indicate "locking points," i.e., locations corresponding to the same value of $x \forall t$ (cf. Remark 4.5.5). Finally, note that, as in Figure 4, those portions of the invariants that coincide in the limit $\epsilon \rightarrow 0^{+}$can be omitted when calculating the local genus.

(ii) $q_{0}<u_{0}<2 q_{0}$ :

This case is regularized by the genus-4 NLS-Whitham equations (i.e., with ten invariants), as illustrated in Figure $6 \mathrm{c}, \mathrm{d}$, where the original and regularized Riemann invariants are shown at two different values of $t$. 


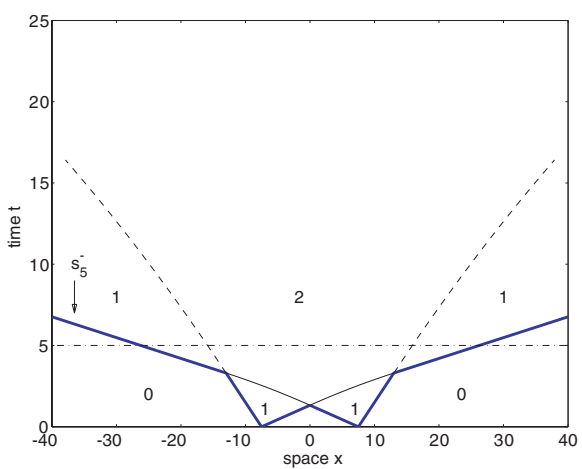

(a)

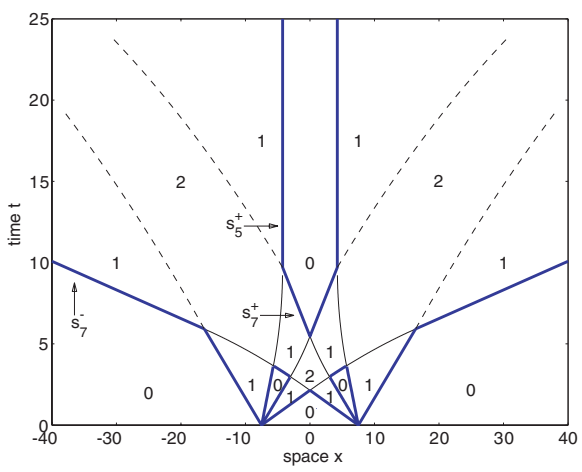

(c)

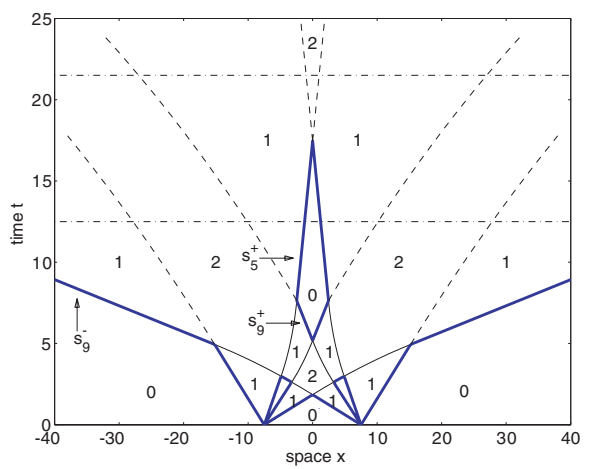

(b)

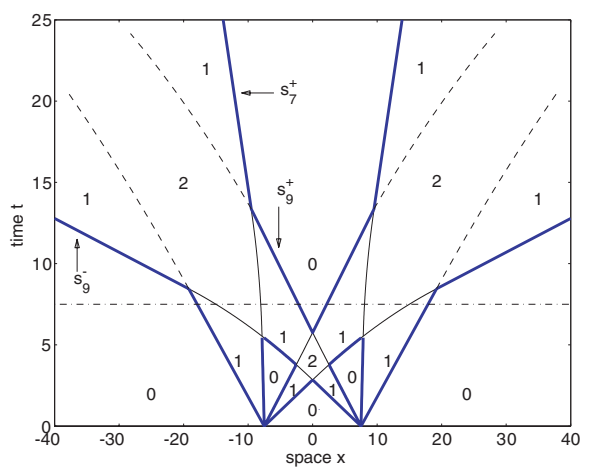

(d)

Fig. 7. Bifurcation diagrams illustrating the regions of genus-0, genus-1, and genus- 2 in the $(x, t)$ plane for the cases shown in Figure 6, namely: (a) $u_{0}=2001 / 1000\left(u_{0}>2 q_{0}\right)$, corresponding to case (i) and Figure 6a; (b) $u_{0}=5 / 4\left(2 q_{0}>u_{0}>q_{0}\right)$, corresponding to case (ii) and Figure $6 \mathrm{c}$,d; (c) the critical case $u_{0}=1$, which is the separatrix between cases (ii) and (iii); and (d) $u_{0}=$ $5 / 8\left(q_{0}>u_{0}>0\right)$, corresponding to case (iii) and Figure 6b. The horizontal axis is position, $x$, and the vertical axis is time, $t$. In all cases, $q_{0}=1$. The thick lines indicate the boundaries between regions of genus- 0 and genus- 1 (cf. Propositions 4.4, 4.7, and 4.8). The thin lines indicate boundaries between genus- 1 and genus- 2 regions, and were computed by approximating them with circular arcs of given starting point and given initial and final slopes. (These conditions determine the circular arc uniquely.) The thin dashed lines indicate boundaries between genus- 1 and genus- 2 regions in those cases when the final slope is unknown. The dot-dashed horizontal lines indicate the values of $t$ corresponding to the diagrams of the Riemann invariants in Figure 6a-d.

The regions of genus- 0,1 , and 2 for the solution of the NLS equation are shown in the bifurcation diagram in Figure 7b. Both jumps are subcritical individually, so two genus-0 regions open up initially at the location of each frequency jump (each surrounded by genus-1 regions, as in case (ii) of Section 3). As these two portions of the solution interact, a genus- 0 region forms temporarily in the central portion of the solution. This region disappears after a while, however, and a genus- 2 region forms which expands forever as in the previous case (cf. Propositions 4.4, 4.7, and 
4.8). In terms of the numerical simulations, this case corresponds to Figure $11 \mathrm{~b}$ and Figure $13 \mathrm{c}, \mathrm{d}$.

(iii) $0<u_{0}<q_{0}$ :

This case is also regularized by the genus-4 NLS-Whitham equations. The original and regularized Riemann invariants are shown in Figure 6b, and the regions of genus- 0,1 , and 2 for the solution of the NLS equation are shown in the bifurcation diagram in Figure 7 d.

As in the previous case, the individual jumps are subcritical, so two genus- 0 regions open up initially at the location of each frequency jump. Also similarly to the previous case, a genus- 0 region forms in the central portion of the solution as the two genus- 0 portions of the solution interact. Contrary to the previous case, however, the genus- 0 portion of the solution now persists and expands forever (cf. Propositions 4.4, 4.7, and 4.8). Numerical simulations corresponding to this case are shown in Figure 11c and Figure 13a,b.

(iv) $u_{0}<0$ :

As in the single-jump case, two expanding depression regions form at the location of each frequency jump. Eventually, these regions merge to form a unique depression zone. As in the single-jump case, the details of the regularization process vary depending on the value of $u_{0}$. Because the solution is always genus- 0 , however, this case is not as interesting as the previous ones in terms of applications, and therefore it will not be investigated further.

Let us now discuss more quantitatively the behavior of the solutions in each of the above scenarios. When $u_{0}>0$ as in cases (i)-(iii), the initial values of the nonregularized Riemann invariants $r_{1,2}^{0}(x)$ are increasing functions of $x$, and one has

$$
\begin{aligned}
& \max _{x \in \mathbb{R}} r_{1}^{0}(x)=r_{1}^{0}(\infty)=\lim _{x \rightarrow \infty} r_{1}^{0}(x)=2\left(u_{0}-q_{0}\right), \\
& \min _{x \in \mathbb{R}} r_{2}^{0}(x)=r_{2}^{0}(-\infty)=\lim _{x \rightarrow-\infty} r_{2}^{0}(x)=-2\left(u_{0}-q_{0}\right)
\end{aligned}
$$

As we will see in Section 6, cases (ii) and (iii) offer the most useful behavior for practical applications. In particular, the case $u_{0}=q_{0}$, which is the separatrix between cases (ii) and (iii), is especially interesting since it produces a high-amplitude genus- 0 region of constant width at the center of the pulse, as shown in Figure 7c. This case is regularized by the genus-3 NLS-Whitham equations, corresponding to Figure $6 \mathrm{~b}$ with $r_{5}$ and $r_{6}$ deleted and where now $r_{1}^{0}(\infty)=r_{2}(-\infty)$, since $u_{0}=q_{0}$. Hereafter, we will call the case $u_{0}=q_{0}$ the "critical" two-jump case, and we will refer to cases (ii) and (iii) as the supercritical and subcritical two-jump cases, respectively. More precisely,

Definition 4.2. We say that an arbitrary collection of positive frequency jumps $C_{1}, \ldots$, $C_{N}$ located at positions $X_{1}, \ldots, X_{N}$ is collectively supercritical if $r_{1}^{0}(\infty)>r_{2}^{0}(-\infty)$, collectively subcritical if $r_{1}^{0}(\infty)<r_{2}^{0}(-\infty)$, and collectively critical if $r_{1}^{0}(\infty)=$ $r_{2}^{0}(-\infty)$, where as usual $r_{k}^{0}( \pm \infty)=\lim _{x \rightarrow \pm \infty} r_{k}^{0}(x)$.

Note the difference between the above and Definition 3.1, which distinguishes whether a single jump is individually subcritical or supercritical. 
It should be noted that, even though the NLS-Whitham equations with $g=3,4$ are necessary to regularize the data in some cases, only the genus $g \leq 2$ appears in the solution with two frequency jumps (similar to the scenario with a single frequency jump). Note also that, unlike the case when a single phase is present, calculating analytically the precise location of the boundaries between different multiphase regions after the individual portions of the solutions have come into contact is a highly nontrivial task, and we have not attempted to do so. However, it is possible to calculate the velocity of all boundaries between genus- 0 and genus- 1 regions. The main reason for the difference between the two types of boundaries is that, upon removal of degenerate gaps, the calculation of characteristic speeds in regions of genus- 1 is expressed in terms of elliptic integrals and the boundary with genus-0 turns out to be a straight line (see Lemma 4.3 below), whereas the characteristic speeds in regions of genus- 2 require the evaluation of hyperelliptic integrals (e.g., see [19]).

The explicit calculation of a few characteristic speeds is reported in Appendix A.4. Since such calculations are rather lengthy, however, and since the methods used are described in [7], [8], [27], here we omit the details relative to all the different cases, and we limit ourselves to summarizing the main results.

Lemma 4.3. All boundaries between genus-O and genus-1 regions are given by straight lines.

Proof. The result follows from (2.3) and by noting that the characteristic speeds are constant since all the invariants are constant in the genus- 0 portion.

This result is obviously independent of the number of frequency jumps present, and is just a special case of a generic feature of hyperbolic systems: If one side of the initial condition is constant, there is a solution in terms of simple waves, for which the characteristics are straight lines [36].

Proposition 4.4. The boundaries between genus-0 and genus-1 regions before the interaction are given by straight lines with the same velocities as in (3.1) and (3.2) upon application of the appropriate Galilean shifts. That is, $s_{i, \pm}= \pm s_{i}+u_{0}$ for the regions relative to the frequency jump to the left and $s_{i, \pm}= \pm s_{i}-u_{0}$ for those relative to the frequency jump to the right.

Proof. The result follows by direct computation of the characteristic speeds in the NLSWhitham equations (2.3), but is consistent with Lemma 4.1 regarding the application of a constant offset to a single frequency jump, showing that before interaction the behavior of the solution in each region is unaffected by the presence of the other jump.

Remark 4.5. In case (ii) (cf. Figure 6c, d and Figure 7b), it is $r_{5}\left(x_{*}, t\right)=r_{6}\left(x_{*}, t\right)=$ $r_{7}\left(x_{*}, t\right)$ for all $t$, where $x_{*}(t)=\max \left\{x \in \mathbb{R}: r_{5}(x, t)=2\left(u_{0}-q_{0}\right)\right\}$. That is, the three invariants are "locked" together at that point. Similarly, if one defines $x_{* *}(t)=\min \{x \in$ $\left.\mathbb{R}: r_{6}(x, t)=-2\left(u_{0}-q_{0}\right)\right\}$, it is $r_{4}\left(x_{* *}, t\right)=r_{5}\left(x_{* *}, t\right)=r_{6}\left(x_{* *}, t\right)$ for all $t$. This locking phenomenon is identified by the two ellipses in Figure $6 \mathrm{c}, \mathrm{d}$ (see also Figure $2 \mathrm{a}$ ). 
Moreover, since two of the three invariants always coincide in a neighborhood of $x_{*}$ or $x_{* *}$, in each case we obtain a trivial regularization locally. That is, near $x=x_{*}$, the three Riemann invariants $r_{5}, r_{6}$, and $r_{7}$ are reduced to a single invariant $\hat{r}(x, t)$ given by $\hat{r}(x, t)=r_{7}(x, t)$ for $x<x_{*}(t)$ and $\hat{r}(x, t)=r_{5}(x, t)$ for $x>x_{*}(t)$, and a similar situation arises for $r_{4}, r_{5}$, and $r_{6}$ near $x=x_{* *}$. Those trivial regularizations are, however, necessary in order for all the Riemann invariants to be nonincreasing functions in $x$ (unlike the case in Figure 2a, where trivial ones can be removed).

Conjecture 4.6. The width of the outermost genus-2 regions present in cases (ii) and (iii) after interaction tends asymptotically to zero.

In both case (ii) and case (iii), the speeds of the left- and right-boundaries of the genus- 2 region in question are respectively given by $s_{9}^{+}$and $s_{7}^{-}$(cf. Figures 4 and 7), where as before the superscripts "-" and "+" refer to the values of the Riemann invariants respectively to the left and to the right of their discontinuities at $t=0$. The fact that the width decreases monotonically and tends asymptotically to a constant follows from the double sorting property of the characteristic velocities. It is not possible, however, to prove whether the asymptotic width is zero without explicitly calculating the speeds of the boundaries between genus- 1 and genus- 2 regions, which is beyond the scope of this work. (The appearance of this phenomenon was first noted by F.-R. Tian for the case of the KdV-Whitham equations with two jumps [32].)

Proposition 4.7. After interaction, the boundary between the outermost genus-1 regions and the surrounding genus-0 regions is given by $x= \pm\left(x_{0}+s_{\text {outer }} t\right)$, where $s_{\text {outer }}=s_{5}^{-}$ in case ( $i$ ) and $s_{\text {outer }}=s_{9}^{-}$in cases (ii) and (iii). In all these cases, the resulting velocity is

$$
s_{\text {outer }}=\left(2 u_{0}+q_{0}\right)\left[1+2 u_{0} q_{0} /\left(2 u_{0}+q_{0}\right)^{2}\right] .
$$

The characteristic speed can be obtained from degenerate $g=2$ calculations (cf. Appendix A.4). Note, however, that the appropriate values of $s_{5}^{-}$and $s_{9}^{-}$can also be obtained from $s_{3}^{+}$in (3.1) and $s_{5}^{+}$in (3.2) upon $u_{0} \rightarrow 2 u_{0}$. (Cf. Figures 4 and 6, and discard the Riemann invariants corresponding to degenerate gaps.)

Proposition 4.8. After interaction, the boundary between the innermost genus-0 region and the surrouding genus-1 regions in cases (ii) and (iii) can be written as

$$
x= \pm\left(x_{1}+s_{\text {inner }}^{(1)} t\right), \quad x= \pm\left(x_{2}+s_{\text {inner }}^{(2)} t\right),
$$

respectively for the initial portion and for the final portion, where the characteristic speeds are: $s_{\text {inner }}^{(1)}=s_{9}^{+}$both in case (ii) and case (iii); $s_{\text {inner }}^{(2)}=s_{5}^{+}$in case (ii), and $s_{\text {inner }}^{(2)}=s_{7}^{+}$in case (iii). Furthermore, in both case (ii) and case (iii), the values of these speeds are

$$
s_{\text {inner }}^{(1)}=q_{0}, \quad s_{\text {inner }}^{(2)}=q_{0}-u_{0} .
$$


As before, the characteristic speeds can be obtained via degenerate $g=2$ calculations (see Appendix A.4 for more details). As before, however, the appropriate values of $s_{5}^{+}, s_{7}^{+}$, and $s_{9}^{+}$can also be obtained from $s_{5}^{+}$in case (ii) of Section 3 upon rescaling $q_{0} \rightarrow q_{0}+\frac{1}{2} u_{0}$ and $u_{0} \rightarrow 2 u_{0}$. More precisely, neglecting degenerate gaps, the Riemann invariants that determine the value of $s_{\text {inner }}^{(1)}=s_{5}^{+}$in case (ii) and $s_{\text {inner }}^{(1)}=s_{7}^{+}$in case (iii) are obtained from those that determine $s_{5}^{+}$in Figure $4 \mathrm{~b}$ upon rescaling $u_{0} \rightarrow 2 u_{0}$. (Cf. Figures $4 \mathrm{~b}$ and 6b,c.) Similarly, the Riemann invariants that determine the value of $s_{\text {inner }}^{(1)}=s_{9}^{+}$both in case (ii) and in case (iii) are obtained from those that determine $s_{5}^{+}$in Figure $4 \mathrm{~b}$ (case (ii) in Section 3) upon rescaling $q_{0} \rightarrow q_{0}+\frac{1}{2} u_{0}$. (Again, cf. Figures $4 \mathrm{~b}$ and 6b,c.)

Remark 4.9. Equations (4.5) and (4.7) also describe the motion of the boundaries of the genus- 0 regions in the critical case $u_{0}=q_{0}$, upon proper relabeling of the Riemann invariants. In particular, they predict that in the critical case the width of the inner genus- 0 region is constant (i.e., $s_{\text {inner }}^{(2)}=0$ ).

The above results, and in particular (4.5) and (4.7), show that the speed of the boundaries between regions of genus- 0 and genus- 1 after the individual perturbations have come into contact is significantly altered as a result of the interaction. In other words, the interaction between different genus- 1 regions results in significant bending. This can perhaps be best appreciated in the critical case (i.e., $u_{0}=q_{0}$, cf. Figure 7c).

Finally, we note that, as in the case of a one-frequency jump, all of the above values for the speeds of the boundaries between genus- 0 and genus- 1 regions agree very well with the results of numerical simulations of the NLS equation, to be discussed in Section 6 .

\section{Behavior of Finite-Genus Solutions: Arbitrary Number of Jumps}

Similar calculations to the ones described in Section 4 can be repeated for jumps of arbitrary size and/or when more than two frequency jumps are present. In particular, from the generalization of the above calculations to an arbitrary number of jumps, it is possible to extract some general features. Thus, we consider an initial condition with constant-amplitude and with initial frequency expressed in terms of $N$ frequency jumps each of size $C_{j}$ and located at $x=X_{j}, j=1, \ldots, N$ :

$$
u(x, 0)=-C_{\mathrm{tot}} / 2+\sum_{j=1}^{k} C_{j}, \quad \text { for } \quad X_{k}<x<X_{k+1},
$$

where $X_{0}=-\infty$ and $X_{N+1}=\infty$, and where $C_{\text {tot }}=\sum_{j=1}^{N} C_{j}=u(\infty, 0)-u(-\infty, 0)$ is the total jump size. The constant frequency offset $C_{\text {tot }} / 2$ in $u(x, 0)$ does not affect the qualitative behavior of solution, and is chosen so that the mean frequency of the solution is zero, i.e., so that the mean position of the solution is constant.

For simplicity, hereafter we will limit ourselves to considering a symmetric collection of positive frequency jumps. That is, we will assume that $C_{j}>0 \forall j=1, \ldots, N$ and that if a given jump of amplitude $C=C_{*}$ exists at $x=X_{*}$, another jump with the same amplitude exists at $x=-X_{*}$. (Or, in other words, the initial condition $u(x, 0)$ for the frequency possesses reflection symmetry with respect to the origin.) Since $C_{j}>0$, 
$\forall j=1, \ldots, N$, the analogue of (4.4) holds:

$$
\begin{aligned}
& \max _{x \in \mathbb{R}} r_{1}^{0}(x)=r_{1}^{0}(\infty)=\lim _{x \rightarrow \infty} r_{1}^{0}(x)=-2 q_{0}-C_{\mathrm{tot}} / 2, \\
& \min _{x \in \mathbb{R}} r_{2}^{0}(x)=r_{2}^{0}(-\infty)=\lim _{x \rightarrow-\infty} r_{2}^{0}(x)=2 q_{0}+C_{\mathrm{tot}} / 2
\end{aligned}
$$

Note that, again, $r_{1}^{0}(x)$ and $r_{2}^{0}(x)$ are the Riemann invariants before regularization. Owing to (5.2) and Definition 4.2, a collection of jumps will be supercritical if $C_{\text {tot }}>2 q_{0}$, subcritical if $C_{\text {tot }}<2 q_{0}$, and critical if $C_{\text {tot }}=2 q_{0}$.

Proposition 5.1. In the case of $N$ equal frequency jumps each of size $C=2 u_{0}$, no individual genus-O regions develop if the jumps are individually supercritical, i.e., $u_{0}>2 q_{0}$.

Recall that the size of each of the jumps is $2 u_{0}$ and that the jumps are individually supercritical according to Definition 3.1 when the original Riemann invariants overlap at a single location in space, that is, if $r_{2}^{0}\left(X_{j}^{-}\right)<r_{1}^{0}\left(X_{j}^{+}\right)$for $j=1, \ldots, N$. The result applies independently of the number of jumps. More in general, however, for an arbitrary collection of frequency jumps we have the following:

Theorem 5.2. It is possible to obtain arbitrarily large genera for finite times by considering appropriate collections of frequency jumps. However, asymptotically as $t \rightarrow \infty$, the only expanding regions in the solution are of genus-0, genus-1, and genus-2.

Proof. The result follows directly from Proposition 2.1 (double sorting property of the characteristic speeds). Let us first note that the nonregularized system has two Riemann invariants, and therefore, independently of the behavior at intermediate values of $x$, the regularized system will have only two nondegenerate branches as $x \rightarrow \pm \infty$, i.e., $r_{1}^{0}(x)<r_{2}^{0}(x)$. The only topologically distinct ways to connect these two branches are shown in Figure 8 (some of these possibilities were also studied in [12], but not for genus- 2 cases), where we have neglected the possible presence of one or more shrinking regions such as the outermost genus- 2 regions in Figure $6 \mathrm{~d}$ (see Remark 5.4, later). The distinction between the different cases in Figure 8 is based on the size of the frequency jumps across the pulse. More precisely:

(i) Figure $8 \mathrm{a}$ is obtained when $r_{1}^{0}(\infty)<r_{2}^{0}(-\infty)$, i.e., when the jumps are collectively subcritical (as in Figures $4 b$ and $6 b$ ).

(ii) Figure $8 \mathrm{~b}$ is obtained when $r_{1}^{0}(\infty)>r_{2}^{0}(-\infty)$ but $r_{1}^{0}\left(x^{+}\right)<r_{2}^{0}\left(x^{-}\right) \forall x \in \mathbb{R}$; that is, when the jumps are collectively supercritical, but every jump is individually subcritical (as in Figure 4d).

(iii) Figure $8 \mathrm{c}$ is obtained when there is only one jump, but that jump is supercritical; that is, a single jump at $x=t_{*}$, such that $r_{1}^{0}\left(x_{*}^{+}\right)>r_{2}^{0}\left(x_{*}^{-}\right)$(as in Figure 4a).

(iv) Figure $8 \mathrm{~d}$ is obtained when there is one supercritical jump at the center, surrounded by other jumps which are all subcritical. That is, when there is one jump at $x=0$ such that $r_{1}^{0}\left(0^{+}\right)>r_{2}^{0}\left(0^{-}\right)$, surrounded by other jumps at $x=t_{j}$ such that $r_{1}^{0}\left(x_{j}^{+}\right)<$ $r_{2}^{0}\left(x_{j}^{-}\right) \forall x_{j} \neq 0$. 


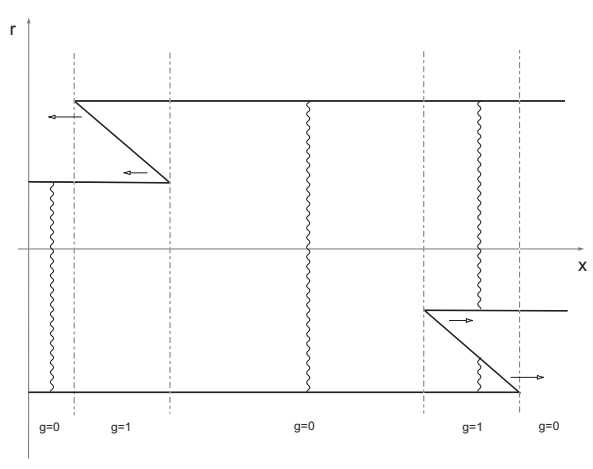

(a)

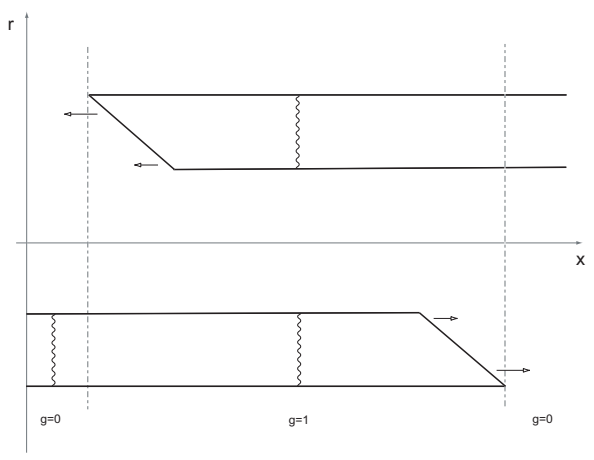

(c)

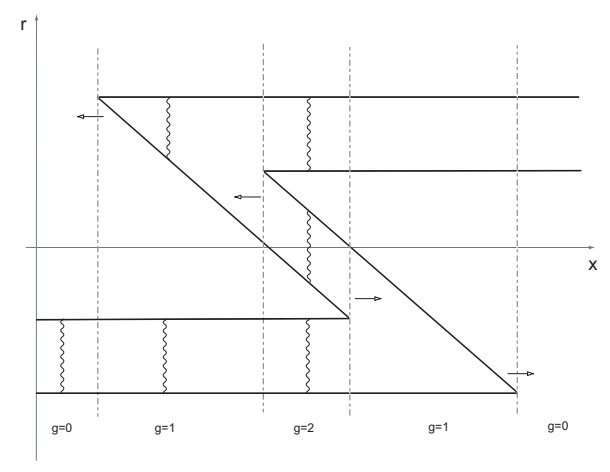

(b)

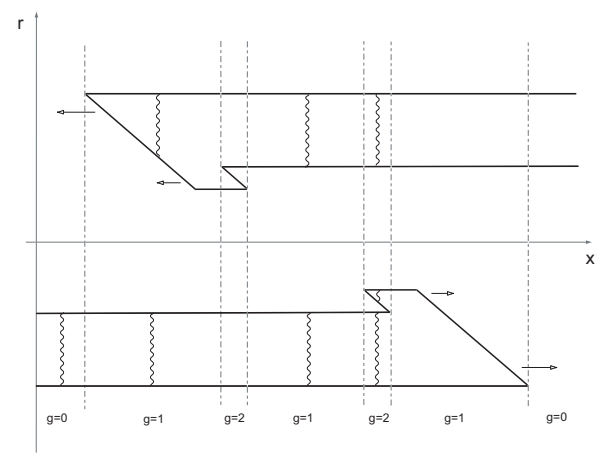

(d)

Fig. 8. Qualitative diagrams illustrating topologically distinct cases for the asymptotic values of the Riemann invariants after removal of the degenerate portions, together with the corresponding separation between regions of genus- 0 , genus- 1 , and genus-2: (a) genus- 0 region at the center, corresponding to Figures $4 \mathrm{~b}$ and $6 \mathrm{~b}$; (b) genus- 2 region at the center, corresponding to Figure $6 \mathrm{~d}$; (c) genus-1 region at the center, corresponding to Figure 4a; and (d) originated by a supercritical jump surrounded by subcritical ones. In each case, the wavy lines show, for some values of $x$, the branch cuts in the Riemann surface $y^{2}=\prod_{j=1}^{2 g+2}\left(z-r_{j}(x, t)\right)$, that is, the regions $\left(r_{2 k-1}, r_{2 k}\right)$ for $k=1, \ldots, g+1$. The gaps in the local spectrum of the Lax operator $\mathcal{L}$ of the NLS equation are the bounded intervals $\left[r_{2 k}, r_{2 k+1}\right]$ for $k=1, \ldots, g$ (cf. Section 2).

Of course, many variations of these basic configurations are possible depending on the details of the arrangement of the frequency jumps. In addition, cases (iii) and (iv) (corresponding to Figure 8c,d), admit a variant in which one or more localized "islands" exist between the two outer portions, as shown in Figure 9a,b (cf. Figure 6a). One of these islands is obtained whenever two additional frequency jumps are inserted that are both individually supercritical, that is, when $r_{1}^{0}\left(x_{*}^{+}\right)>r_{2}^{0}\left(x_{*}^{-}\right)$for more than one value of $x_{*} \in \mathbb{R}$. For example, Figure 9a is produced when there are four supercritical jumps (cf. Proposition 5.1); Figure 9b is produced when there are two supercritical jumps surrounded by two subcritical ones. From these examples it should be clear that the above situations can easily be generalized to cases where an arbitrary number of islands 


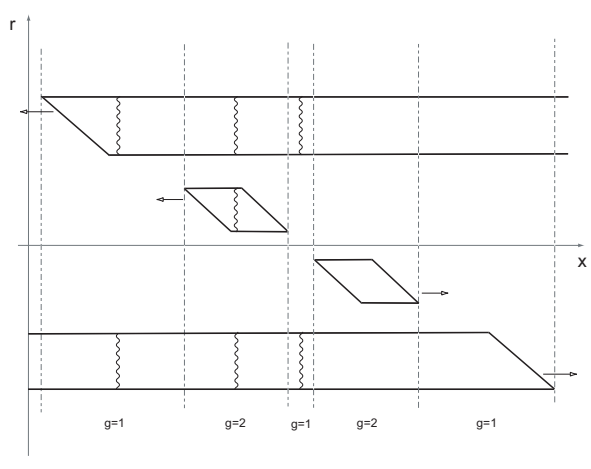

(a)

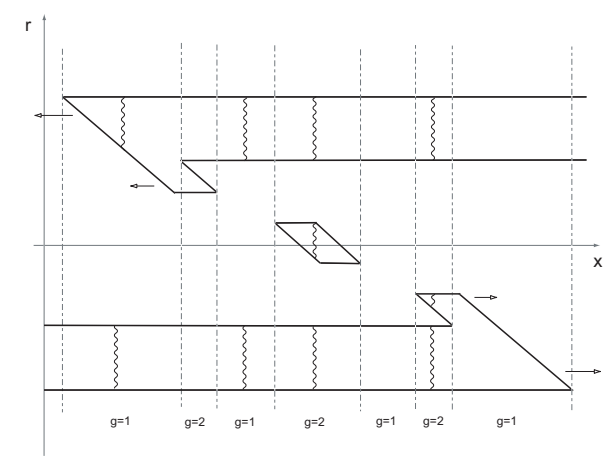

(b)

Fig. 9. Additional diagrams of asymptotic configurations of Riemann invariants, illustrating the possible presence of separate "islands," each of which produces one additional region of genus-2. (a) A variant of Figure 8c corresponding to four supercritical jumps. (b) A variant of Figure 8d corresponding to two supercritical jumps surrounded by two subcritical ones.

is present by adding a proper number of supercritical frequency jumps. Note that the range of values for the Riemann invariants covered by each of these islands is disjoint from that of all of the other islands. (That is, the islands are not lying side by side. Rather, they are arranged by increasing values of the invariants.) Thanks to the double sorting property possessed by the Riemann invariants (namely, (2.4) in Proposition 2.1), islands characterized by larger values of the invariants propagate faster to the left, and therefore, if several islands exist, different islands will temporarily end up stacked on top of each other upon propagation. It is then clear that by properly choosing the number and size of frequency jumps, it is possible to produce regions of arbitrarily high genus for finite values of $t$. However, the sorting property of the Riemann invariants also implies that, eventually, each of the islands will separate from the others. Thus, asymptotically in $t$, at most one island will be present for each value of $x$, and correspondingly there will be at most two gaps (i.e., bounded intervals of the spectrum).

Remark 5.3. The above decomposition of the solution of the NLS equation (1.1) into regions of genus- 0,1 , and 2 asymptotically with time should be compared to the corresponding result for the Korteweg-de Vries (KdV) equation $u_{t}+6 u u_{x}+u_{x x x}=0$, for which it was shown that the solution decomposes into expanding regions of genus- 0 and genus-1 asymptotically in time [19].

Remark 5.4. It is important to note that regions of genus-3 may also exist in the solution of NLS for all finite values of time. For example, Figure 10a shows the invariants produced by a configuration with two collectively subcritical jumps surrounded by two individually supercritical ones, while Figure 10b shows the invariants produced by a collection of three jumps that are individually subcritical but collectively supercritical. Due to the double sorting property of the Riemann invariants, however, the width of these regions decreases monotonically, and tends to a constant value asymptotically with time. Therefore, the presence of these regions does not invalidate the statement of Theorem 5.2. Moreover, 


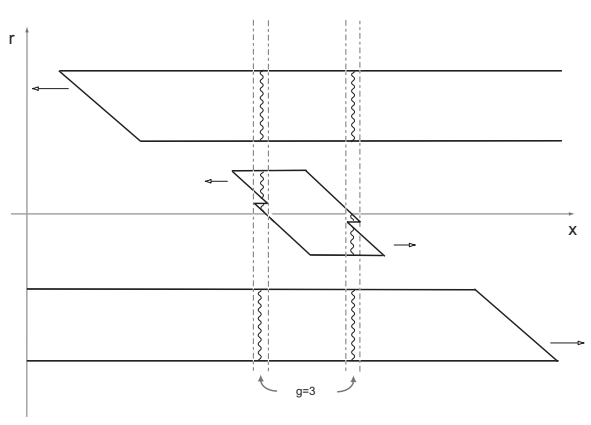

(a)

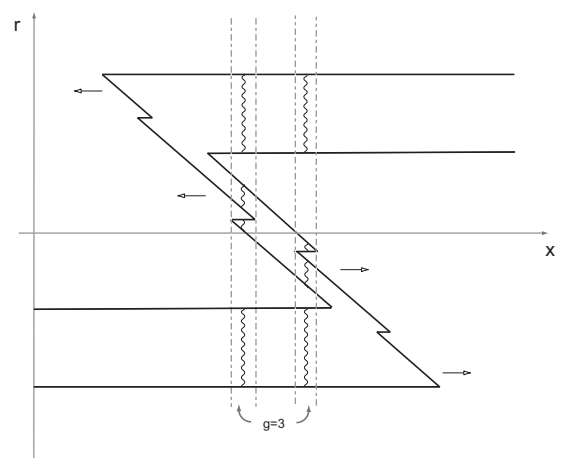

(b)

Fig. 10. Additional diagrams of asymptotic configurations of Riemann invariants showing explicitly the presence of shrinking genus-3 regions; (a) is the analogue of Figure 9b; (b) is the analogue of Figure 8b (see text for details).

we expect the width of these genus-3 regions to tend asymptotically to zero with time (cf. Conjecture 4.6). The situation is similar to the case of the KdV equation, where, in addition to expanding regions of genus- 0 and genus- 1 , shrinking regions of genus- 2 may also exist asymptotically.

From the proof of Theorem 5.2 we also have:

Corollary 5.5. Whenever an overall genus-0 region develops at the center of the pulse as a result of the interaction among all frequency jumps, its amplitude is always given by the analogue of $(1.8)$, namely $\rho_{\max }=\frac{1}{16}\left(r_{2}^{0}(\infty)-r_{1}^{0}(-\infty)\right)^{2}$, in a similar way as for the single-jump case.

In other words, the above value is the largest amplitude of a genus- 0 region that can be achieved with any number $N$ of frequency jumps (cf. Figures $4 \mathrm{~b}$ and $6 \mathrm{~b}, \mathrm{c}$ ). In the case of equal frequency jumps of size $C=2 u_{0}$, it is $\rho_{\max }=q_{0}^{2}\left(1+N u_{0} / 2 q_{0}\right)^{2}$.

Corollary 5.6. Asymptotically as $t \rightarrow \infty$, the solution in the central portion of the pulse will be a region of genus-0 iff the jumps are collectively subcritical, i.e., iff $r_{1}^{0}(\infty)<r_{2}^{0}(-\infty)$

In the case of equal-size frequency jumps, this condition is satisfied iff $u_{0} \leq 2 q_{0} / N$. Thus, the maximum amplitude of a stable genus- 0 region is always $\rho_{\max }=4 q_{0}^{2}$, again as in the single-jump case, and independently of the number of frequency jumps.

All of the results in this work are relative to initial conditions with constant amplitude and piecewise-constant frequency. If the initial amplitude and frequency vary continuously, some differences can obviously be expected in the solution. Because the quasi-linear system of PDEs for the regularized Riemann invariants is always strictly hyperbolic, it is stable with respect to small changes in the initial conditions. Thus, 
even though the previous results are relative to the case of discontinuous amplitude and frequency jumps, they describe generic features of the solution. Consider, for example, the dam-breaking problem. If the amplitude varies continuously from 0 to its maximum value, oscillations appear near the edges of the pulse. (Indeed, these oscillations are visible in the numerical simulations.) In the absence of frequency jumps, this scenario was studied by Forest and McLaughlin using the Lax-Levermore theory [16]. As long as the transition region is narrow, however, the amplitude of these oscillations is small, and therefore the solution will bear a close resemblance to the solutions described here. Similar considerations apply if the frequency transitions are not discontinuous, as will be discussed in Section 6.

The above results are derived assuming a symmetric collection of positive frequency jumps. The presence of negative frequency jumps at the edges or in between other jumps would obviously affect the quantitative details of the picture. We expect that the qualitative features, however, and in particular the asymptotic decomposition into regions of genus-0, 1, and 2, to remain valid.

\section{Behavior of Finite-Genus Solutions: Numerical Simulations}

Even though the analytical calculations described in the previous sections provide a general picture of the behavior of finite genus solutions of the NLS-Whitham equations, there are obviously limits to what can be done analytically. In this section and the following one, we therefore complement those analytical results by presenting numerical simulations of the defocusing NLS equation with small dispersion.

All the numerical results presented are obtained by numerically integrating the NLS equation (1.1) with $\epsilon^{2}=0.1$ using a fourth-order Fourier split-step method. We consider an initial condition $q(x, 0)=|q(x, 0)| \exp [i \varphi(x, 0) / \epsilon]$, where the initial phase $\varphi(x, 0)$ is obtained by integrating (5.1):

$$
\varphi(x, 0)=\sum_{j=1}^{N}\left[-x / 2+\left(x-X_{j}\right) H\left(x-X_{j}\right)\right] C_{j},
$$

where the function $H(x)=1$, if $x \geq 0$, and $H(x)=0$, if $x<0$, represents the Heaviside unit step. The initial pulse amplitude was taken to be a super-Gaussian, namely $|q(x, 0)|=q_{0} e^{-\left(x / \Delta X_{0}\right)^{2 M}}$ with $M=40$. The integration step size and the width $\Delta X_{0}$ of the pulse were chosen to be respectively sufficiently small and sufficiently large that none of the numerical results described in this work are affected by them. In order to remove the factor $\epsilon$ in front of the time derivative in (1.1), the numerical simulations were set up in terms of the fast scale $t^{\prime}=t / \epsilon$. Accordingly, all the numerical results will be described in terms of this time variable. In what follows, we describe the behavior of the solutions for varying values of the parameters $X_{j}$ and $C_{j}$ and for varying number of frequency jumps. We first consider the case of two frequency jumps, to numerically identify regions of different genus (Figure 11) and show a few snapshots of the time evolution (Figure 12). We then proceed to describe three sets of simulations: The first set (Figure 13) shows the effect of gradually increasing the size of the frequency jump. The second set (Figure 14) describes the effect of increasing the number of jumps. Finally, 


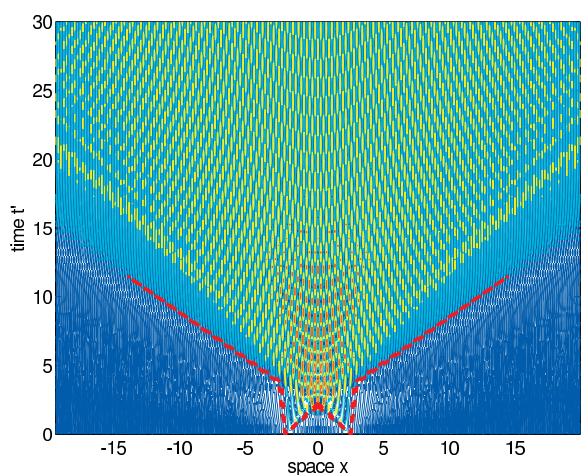

(a)

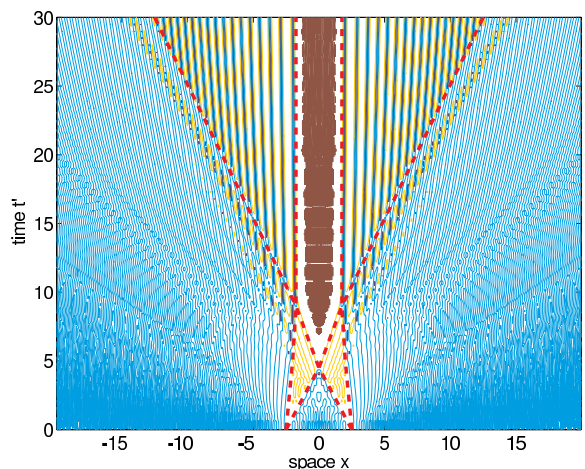

(c)

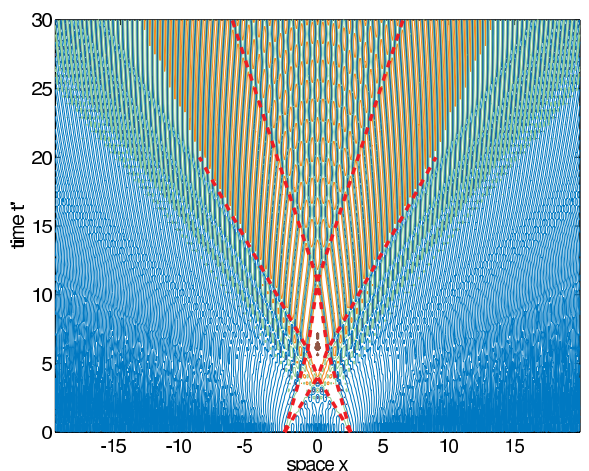

(b)

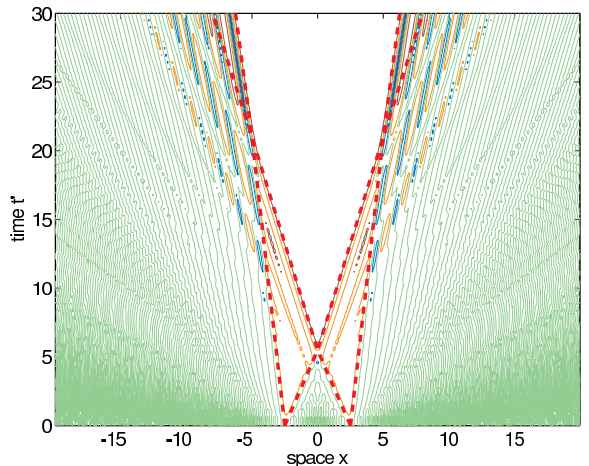

(d)

Fig. 11. Contour plots of numerical simulations of the NLS equation (1.1) for a two-jump initial condition and for different values of the jump size parameter $C$ : (a) $C=5$, corresponding to Figure $6 \mathrm{a}$ and Figure 7a, showing two jumps that are both individually supercritical; (b) $C=3$ corresponding to Figure $6 \mathrm{c}, \mathrm{d}$ and Figure $7 \mathrm{~b}$, showing two jumps which are individually subcritical but supercritical in combination; (c) the case $C=2$, corresponding to Figure 7c in which the two jumps are collectively critical; (d) $C=1$, corresponding to Figure $6 \mathrm{~b}$ and Figure $7 \mathrm{~d}$ and showing a case in which the jumps are collectively subcritical. In each case, the dashed lines demarcate the recognizable regions of different genus. In particular, the speeds of the boundaries between genus- 0 and genus- 1 regions are in very good agreement with the analytical results described in the previous sections.

the third set (Figure 15) shows the effect of changing the initial separation between these jumps. Although numerical experiments were performed with various values of amplitude, all the figures in this work except Figure 18a are relative to the case $q_{0}=1$.

Two frequency jumps. Figure 11 shows contour lines of $|q(x, t)|$ in the $(x, t)$-plane for different values of the jump size $C_{1}=C_{2}=2 u_{0}=: C$. Because of the finite, nonzero value of $\epsilon$, not all of the features presented in the previous section are immediately apparent. In other words, because of the finite value of $\epsilon$, the solution of the NLS 


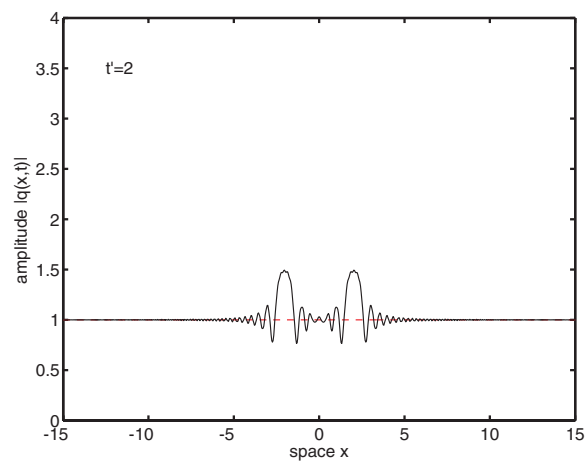

(a)

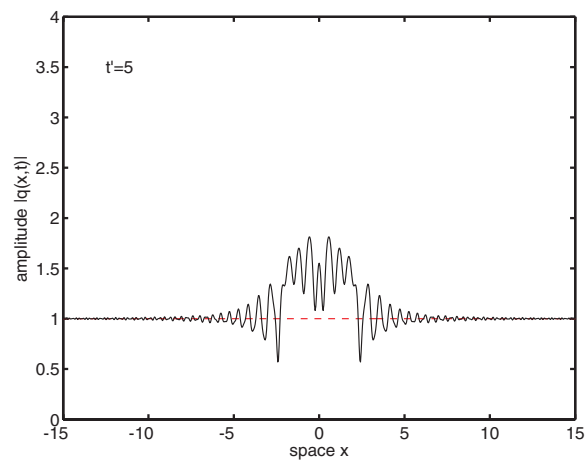

(c)

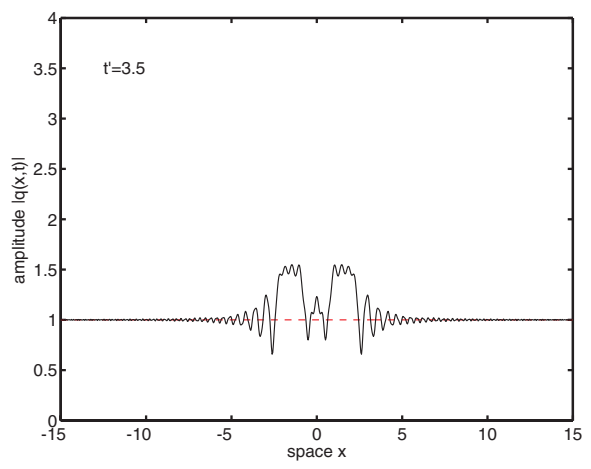

(b)

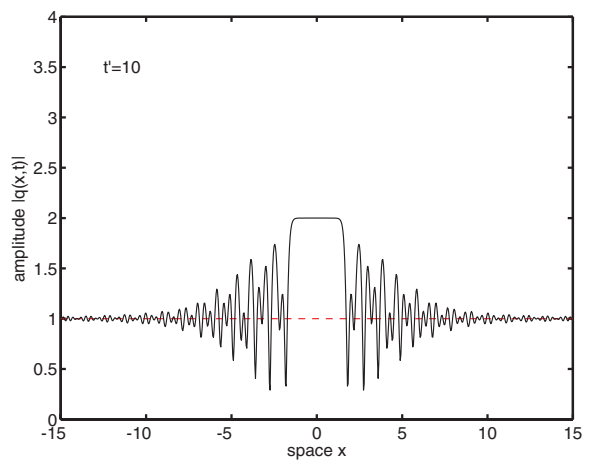

(d)

Fig. 12. Numerical simulations of the NLS equation illustrating the evolution with time of a critical two-jump initial condition. The frequency jumps, located at $X_{2}=-X_{1}=5 / 2$, have amplitude $C_{1}=C_{2}=2$, so that their interaction produces a genus- 0 region of constant width in the center (as shown in Figure 11c). The solution is shown at (a) $t^{\prime}=2$, (b) $t^{\prime}=3.5$, (c) $t^{\prime}=5$, (d) $t^{\prime}=10$. This case corresponds to the contour plot in Figure 11c and the bifurcation diagram in Figure 7c.

equation is only approximately described by a finite-genus solution in each region. Moreover, numerically reconstructing the genus of the solution is a highly nontrivial problem, which is outside the scope of this work. Nonetheless, some regions of genus0 and genus- 1 (whose boundaries have been delimited by dashed lines in Figure 11) are recognizable (e.g., regions of genus- 0 are often flat, and therefore appear white in the contour plots in Figure 11), and their location displays a very good qualitative and quantitative agreement with the analytical calculations presented in Section 4. Note in particular that, even though the value of $\epsilon$ is not particularly small, the value of $C$ which corresponds to the critical case is exactly that which was predicted analytically, namely $C=2$. Figure 12 shows four snapshots illustrating the evolution with time of the critical case shown in Figure 11c. 


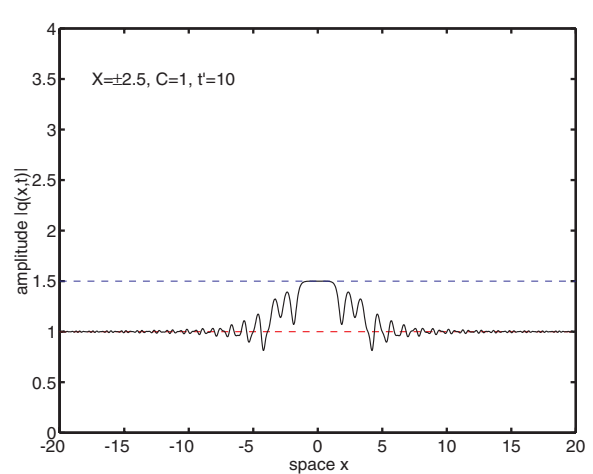

(a)

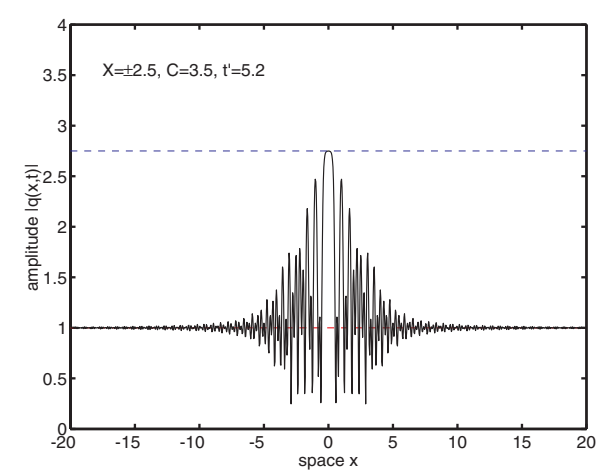

(b)

Fig. 13. Numerical simulations of a two-jump initial condition with $\Delta X=5$, for different values of the jump size $C_{1}=C_{2}=C$ : (a) $C=1$; (b) $C=7 / 2$. The critical case $C=2$ is also shown in Figure 12d. In (a) the solution is subcritical (the genus-0 region expands forever; see Figure $7 \mathrm{~d}$ ), and the solution is shown at $t^{\prime}=10$, whereas in (b) the solution is supercritical (see Figure 7b), and it is shown at the time when the genus- 0 portion has maximum width, $t^{\prime}=5$.2. The horizontal dashed line at $|q|=1$ represents the amplitude of the initial condition, and the other horizontal dashed line identifies the amplitude of the genus-0 region. Note that (i) the amplitude of the genus0 portion of the solution increases linearly with increasing value of $C$, (ii) for increasingly large values of $C$, the genus- 0 solution tends to close more rapidly, and, above a certain threshold, does not open at all.

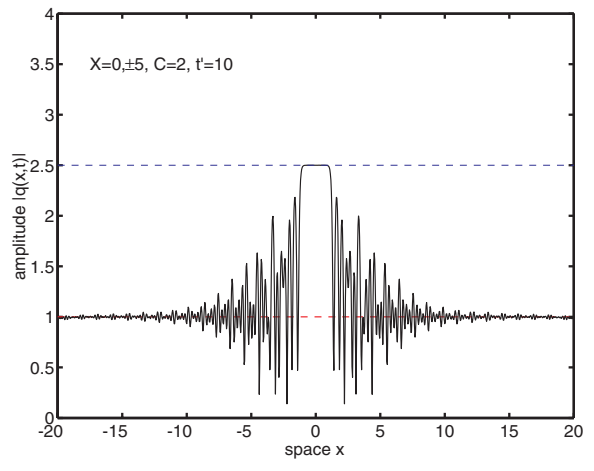

(a)

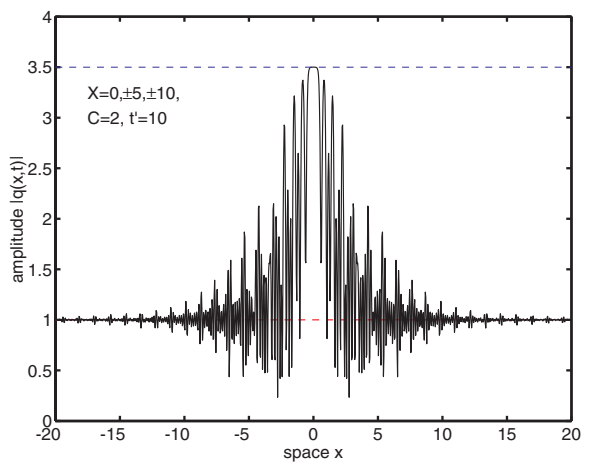

(b)

Fig. 14. Numerical simulations for different numbers $N$ of frequency jumps, with the size of each frequency jump fixed at $C_{i}=2, i=1, \ldots, N$ : (a) $N=3$; (b) $N=5$. In both cases the solution is shown at $t^{\prime}=10$. See also Figure $3 \mathrm{~b}$, illustrating the case $N=1$, and Figure $12 \mathrm{~d}$, illustrating the (critical) case $N=2$. Note that for $N=1$ the central genus- 0 region expands and for $N=2$ it has a steady width, whereas for $N>2$ it exists only for limited times. In all cases the amplitude of this central genus-0 portion increases linearly with the number of jumps. However, for larger number of jumps, this genus- 0 portion tends to close more rapidly, and above a certain number $N$ it does not open at all, at least for a fixed value of $\Delta X$. 


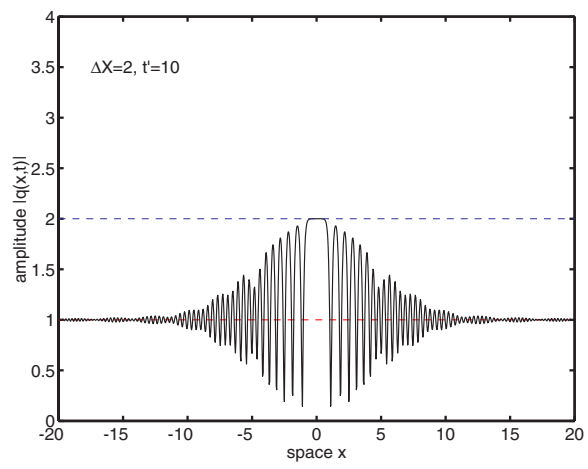

(a)

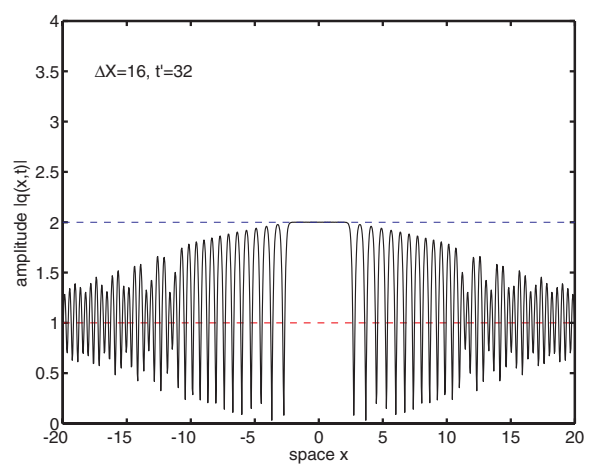

(b)

Fig. 15. Numerical simulations for different separations between jumps, here with a critical arrangement of two frequency jumps of amplitude $C_{1}=C_{2}=2$ : (a) $\Delta X=2$, (b) $\Delta X=16$. See also Figure 12d, which shows the case $\Delta X=5$. In (a) the solution is shown at $t^{\prime}=10$, in (b) at $t^{\prime}=32$. Increasing the value of $\Delta X$ has two main effects: It increases the maximum width of the genus- 0 portion, and it simultaneously lengthens the time scales over which the evolution occurs. See also Figure 7c.

It is also interesting to note that the hexagonal patterns visible in Figure 11 (in particular, near the center $x=0$ in Figures 11a and 11b) show the interaction of two counterpropagating genus-1 (periodic) waves, which produce, as a result, a genus- 2 solution of the NLS equation. Note that in the absence of nonlinearity, a superposition of two simple waves would produce just a parallelogrammic pattern. Thus, the hexagonal pattern is the result of nonlinear phase shifts in the interaction. (Note that the hexagonal pattern can also be found in genus- 2 solutions of the Kadomtsev-Petviashvili equation describing two-dimensional shallow water waves [20].)

Size of the frequency jumps. This set of simulations, some results of which are shown in Figure 13, is relative to a two-jump initial condition. We take the two jumps to be of equal size, $C_{1}=C_{2}=2 u_{0}=C$, and to be initially positioned at $X_{1}=X_{2}= \pm 2.5$. Looking at the behavior of the solution as $C$ varies, one can clearly observe that the amplitude of the genus- 0 portion of the solution increases linearly with increasing value of $C$. More precisely, $|q|_{\max }=q_{0}+C / 2$, in agreement with the analytical calculations presented in the previous section (cf. Corollary 5.5). See also Figure 17a later. Moreover, in a similar way as in the single-jump case, one can see that the maximum amplitude of a stable genus- 0 region is $|q|_{\max }=2 q_{0}$. When $u_{0}$ is larger than the threshold value $q_{0}$, the genus-0 region eventually disappears, as explained in Section 4 and as described in Figure 7b. Unlike the single-jump case, however, one can obtain larger values of $\rho$ for limited times, as shown in Figure 13c,d. Note how, over these shorter times, the amplitude of the genus- 0 region is still given by $|q|_{\max }=q_{0}+C / 2$. Note also, however, that the genus- 0 solution closes more rapidly for increasingly large values of $C$, and above the threshold $C=4$ (i.e., $C=q_{0}$ ) does not open at all, at least for a fixed value of $\Delta X$. These results are in very good agreement with the analytical calculations described in the previous sections (e.g., see Proposition 5.1 and Corollaries 5.5 and 5.6). 
Number of frequency jumps. In this set of simulations, some of which are shown in Figure 14, the number $N$ of frequency jumps was varied, while the size of each jump and their initial spatial separation are kept fixed respectivaly at $C=2$ and $\Delta X=5$. From Figure 14, it is apparent that the amplitude of the genus- 0 part also increases linearly with the number of jumps. More precisely, $|q|_{\max }=q_{0}+N C / 4$, in agreement with the analytical calculations presented in the previous sections (cf. Corollary 5.5). See also Figure $17 \mathrm{~b}$ later. The genus- 0 region, however, will eventually close up if the total jump exceeds the same threshold as in the previous case: $C_{\text {tot }}=N C=4 q_{0}$ (cf. Corollary 5.6). For increasingly larger numbers of jumps, the amplitude of the genus- 0 region follows the same law as above, namely $|q|_{\max }=q_{0}+N C / 4$, and can temporarily achieve very large values. The genus- 0 region also tends to close up more rapidly, however, and, above a certain number of jumps, does not open at all (at least for a fixed value of $\Delta X$ ).

Spatial separation between frequency jumps. In this set of simulations we look at the effect of increasing the spatial separation $\Delta X$ between the frequency jumps. Figure 15 is relative to a two-jump initial condition with $C=2$. The consequences of changes in $\Delta X$ are not as obvious as those of changes in the number of jumps or the jump size. One can see, however, that increasing $\Delta X$ has two main effects: on one hand, it lengthens the time scales over which the evolution occurs, since the various regions need to travel for longer distances to come into contact and to interact; at the same time, it determines the width of the stable genus- 0 region in the critical case. In fact, numerical results show a remarkable fact, namely that the width $X_{\infty}$ of the stable genus- 0 region depends linearly on the initial spatial separation $\Delta X$ between the jumps. In the case of two frequency jumps, one simply has $X_{\infty}=\Delta X / 2$. (Cf. Figures 11c, 12, and 15.) A similar relation, however, holds independently of the number of jumps. For a critical arrangement of an arbitrary number $N$ of equal size, equally spaced positive jumps, it is $X_{\infty}=(N-1) \Delta X / 2$, where $\Delta X$ is the initial separation between consecutive jumps. (Cf. Figure 16, and note that $(N-1) \Delta X$ is just the distance among the farthest two frequency jumps.) These relations are verified in Figure 18a,b.

Summary. Let us recapitulate for convenience the main results of the numerical experiments discussed in the above paragraphs. Given $N$ positive frequency jumps with individual sizes $C_{j}$ and separations $\Delta X_{j}$, we can observe that

(i) The value of the asymptotic genus in the central portion of the pulse is determined only by the total jump $C_{\text {tot }}=C_{1}+\cdots+C_{N}$ (or, equivalently, by the size per jump $C=C_{\mathrm{tot}} / N$ in the case of $N$ jumps of equal size). More precisely, the solution will be genus-0 iff $C_{\text {tot }}<q_{0}$, in very good agreement with the analytical results (see Corollary 5.6). Of course, the detailed behavior of the solution for finite times depends on the precise values of all the $C_{j}$ and $\Delta X_{j}$.

(ii) If a genus- 0 region is desired that expands forever at the center of the pulse, its height is limited by the critical value of the cumulative jump $C_{\text {tot }}$. More precisely, $|q|_{\max }=2 q_{0}$, independently of the number of jumps. For equally sized jumps, this value is obtained for $C=4 q_{0} / N$, in very good agreement with the analytical results (see Corollary 5.5). 


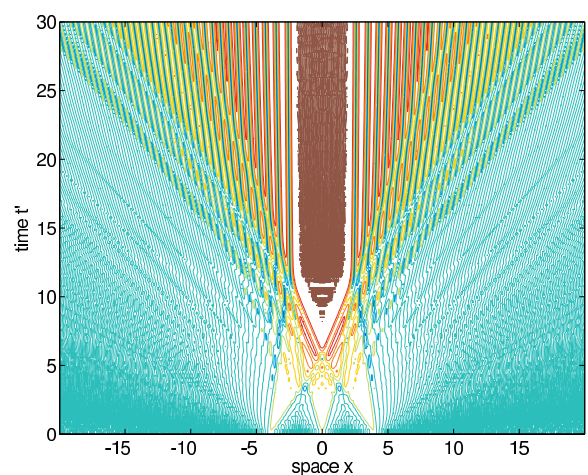

(a)

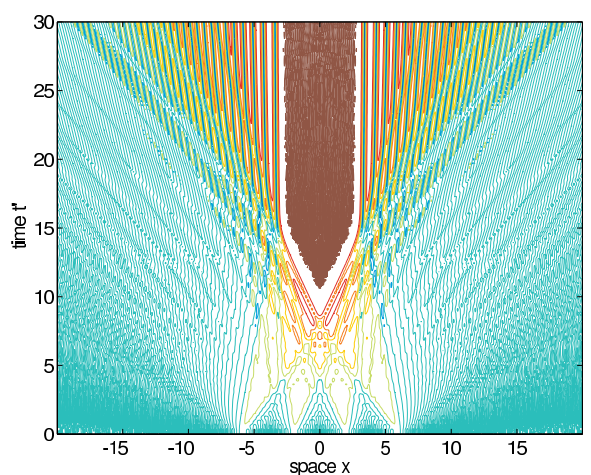

(b)

Fig. 16. Critical arrangements of more than two jumps: (a) $N=3$ and $C=4 / 3$; (b) $N=4$ and $C=1$. In both cases, $q_{0}=1$ and $\Delta X=4$.

(iii) It is possible to achieve genus-0 regions of much larger amplitudes over limited times by employing a sufficiently large number of jumps. More precisely, $|q|_{\max }=$ $q_{0}+C_{\text {tot }} / 4$ (i.e., $|q|_{\max }=q_{0}+N C / 4$ for equally sized jumps), as shown in Figure 17 , and again in very good agreement with the analytical results (see Corollary 5.5).

(iv) For a critical arrangement of an arbitrary number of equally sized, equally spaced positive frequency jumps, the width of the stable genus- 0 region is half of the initial separation between the farthest two frequency jumps. This fact has not been demonstrated analytically, but is nonetheless very solidly supported by the numerical results, as shown in Figure 18.

In the next section we discuss how these results - and in particular items (iii) and (iv) can be used to generate intense, ultra-short optical pulses.

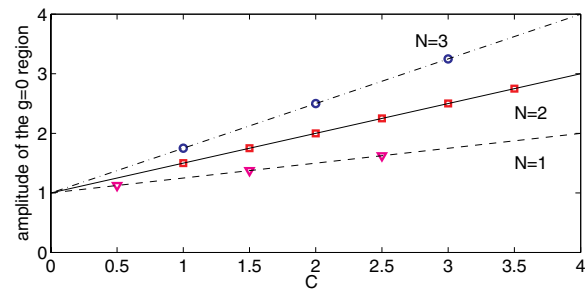

(a)

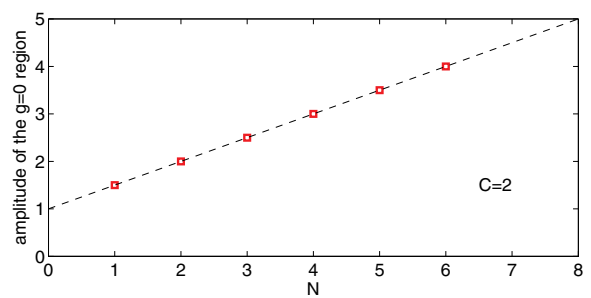

(b)

Fig. 17. (a) The numerically calculated amplitude of the genus- 0 region produced by an arrangement of $N$ equally sized frequency jumps as a function of the individual jump size $C$. Triangles: $N=1$; squares: $N=2$; circles: $N=3$. For comparison, the solid, dashed, and dot-dashed lines show the straight lines $y=(N-1) C / 4$. (b) The amplitude of the genus- 0 region produced by an arrangement of frequency jumps each of size $C=2$, as a function of the number $N$ of jumps. Squares: numerical simulations; dashed line: the line $y=1+N / 2$. 


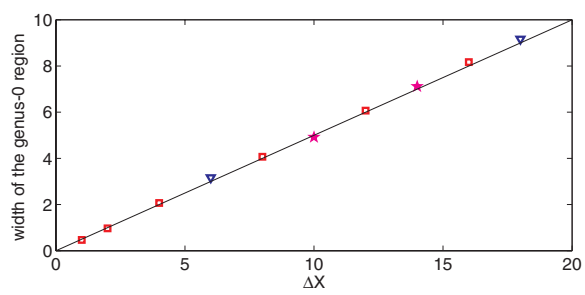

(a)

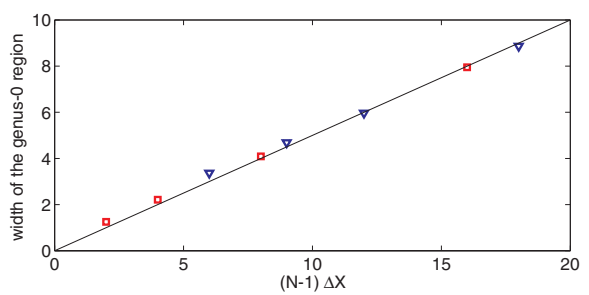

(b)

Fig. 18. Width of the genus- 0 region resulting from the interaction of a critical set of frequency jumps as a function of the spatial separation between them: (a) $N=2$; solid line: the straight line $y=\Delta X / 2$; squares: simulations with $q_{0}=1$ and $C=2$; triangles: simulations with $q_{0}=2$ and $C=4$; stars: simulations with $q_{0}=3$ and $C=6$. (b) $N=3$ (squares) and $N=4$ (triangles), with $q_{0}=1$ in both cases; solid line: the straight line $y=(N-1) \Delta X / 2$. Note that, since the characteristic periodi of the rapid oscillations is $O(\epsilon)$, it is only possible to measure the width of the genus-0 region with precision $\Delta x= \pm \epsilon$.

\section{Applications: Generation of Intense Short Optical Pulses}

The analytical and numerical results described in the previous sections can be used to design an initial condition that results in a genus- 0 region of arbitrarily high amplitude at any desired distance down the fiber by employing a sufficiently long continuous wave $(\mathrm{CW})$ pulse at the outset, as we now describe. (Recall that for fiber optics $x$ is the retarded time and $t$ is the propagation distance along the fiber.) Of course, the same techniques can also be applied in any physical context where the dispersionless limit of the NLS equation is relevant. The basic building block consists of two frequency jumps of critical size, which generate a genus-0 region of constant width, as shown in Figure 11c. By including additional frequency jumps to both sides of this basic pair, one can then temporarily produce an overall genus- 0 region of higher amplitude. This basic process is illustrated in Figure 19, which shows the time evolution of a four-jump initial condition. A contour plot of this solution is shown in Figure 20a. We now discuss some practical issues related to the generation of these high-intensity pulses: namely, how to control their amplitude, the distance along the fiber at which they are produced, their temporal width, and post-processing through filtering in order to eliminate the high-frequency oscillations.

Amplitude, location, and width. By appropriately choosing the number, amplitude and location of the additional frequency jumps, it is possible to precisely specify the amplitude of the overall genus- 0 region as well as to prescribe its width and the distance along the fiber at which it is generated. This is illustrated in Figure 20, which contains contours of four different initial conditions. Figure 20a corresponds to the four-jump solution shown in Figure 19: the two central frequency jumps are critical $\left(C_{2}=C_{3}=2\right)$, while the single, subcritical frequency jumps on either side of them $\left(C_{1}=C_{4}=3\right)$ provide two expanding genus- 0 regions that go to interact with the main one, resulting in an overall genus-0 region of amplitude $|q|_{\max }=q_{0}+C_{\text {tot }} / 4=7 / 2$, once more in perfect agreement with the analytical prediction. 


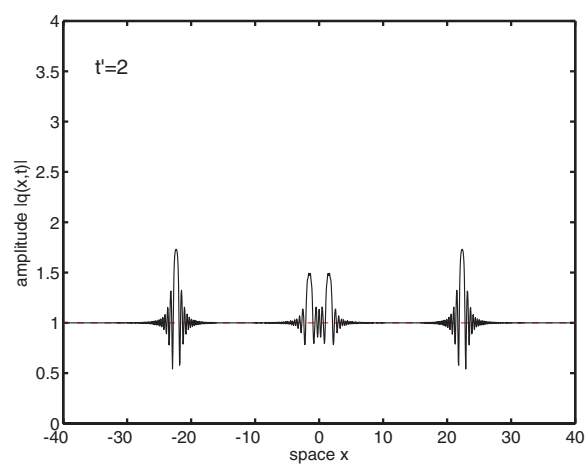

(a)

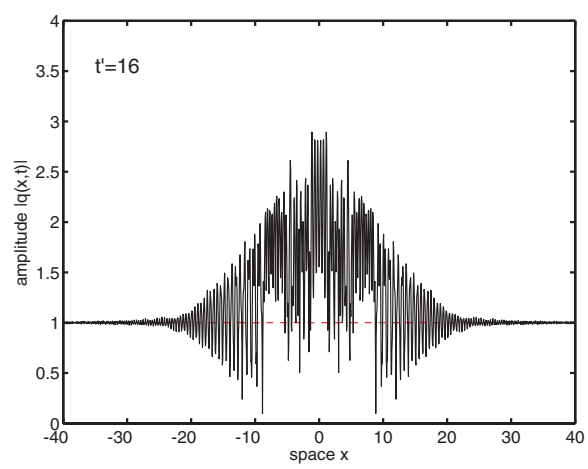

(c)

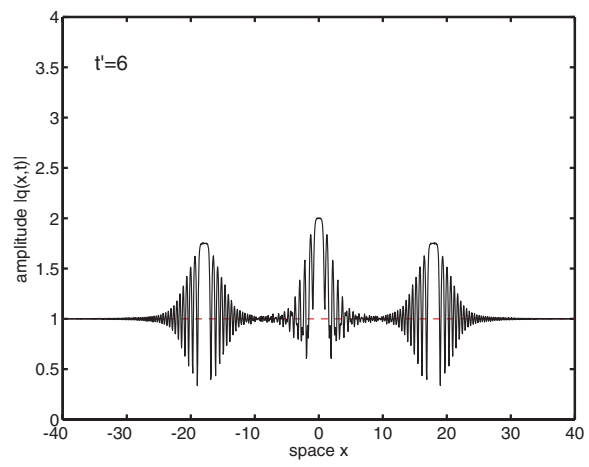

(b)

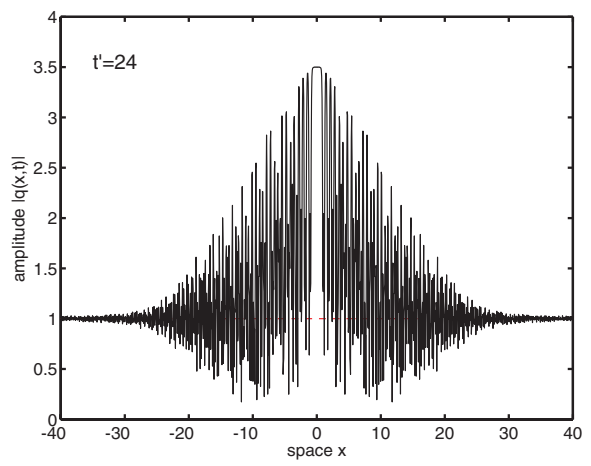

(d)

Fig. 19. Numerical simulations of the NLS equation with a supercritical four-jump initial condition. The frequency jumps, located at $X_{4}=-X_{1}=24$ and $X_{3}=-X_{2}=2$, have amplitude $C_{2}=C_{3}=2$ (so that the interaction of the two center jumps produces a critical genus- 0 region in the center) and $C_{1}=C_{4}=3$, so that the two individual subcritical jumps open up a genus-0 region that moves towards the center of the pulse. The solution is shown at (a) $t^{\prime}=2$, (b) $t^{\prime}=6$, (c) $t^{\prime}=16$, (d) $t^{\prime}=24$. A contour plot of this solution is shown in Figure 20a.

With similar arrangements of four jumps, one can obtain any value of amplitude $|q|_{\max }<4$, at which point the two side jumps become critical and do not generate a genus-0 region anymore. This limitation on the pulse amplitude can be overcome, however, by considering a larger number of frequency jumps. Figures $20 \mathrm{~b}, \mathrm{c}$ are both relative to a six-jump initial condition with all jumps having amplitude $C_{j}=2$, so that each pair of adjacent jumps generates a genus- 0 region of constant width. These regions then interact to create an overall genus- 0 region of amplitude 4 . In Figure 20b, the jump locations $-X_{3}=X_{4}=2,-X_{2}=X_{5}=15,-X_{1}=X_{6}=20$ were chosen so that the overall genus-0 region arises near $t^{\prime}=15$. In Figure 20c, instead, the side jumps $-X_{2}=X_{5}=20,-X_{1}=X_{6}=35$ were positioned further away, so that the genus-0 region emerges near $t^{\prime}=25$ and is maintained for a longer interval. Finally, Figure 20d 


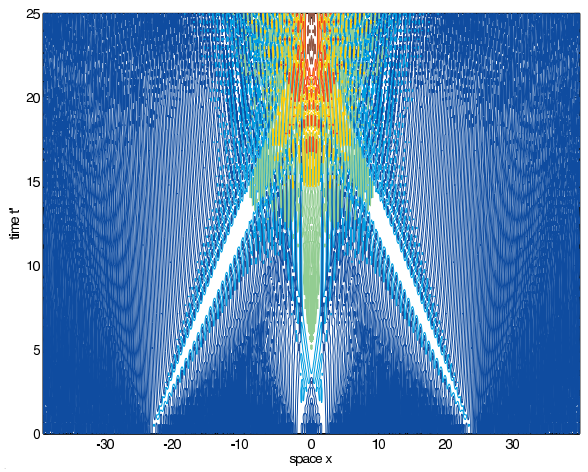

(a)

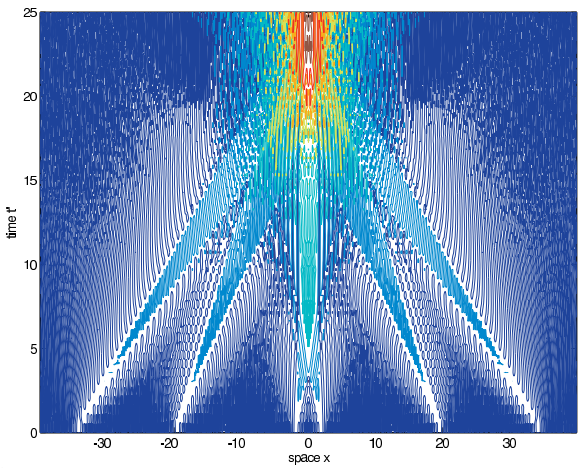

(c)

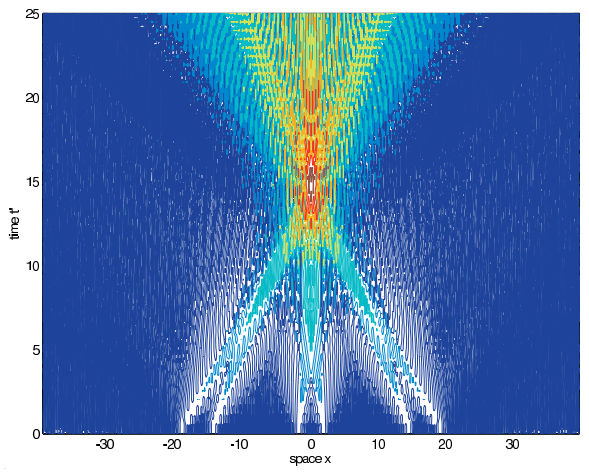

(b)

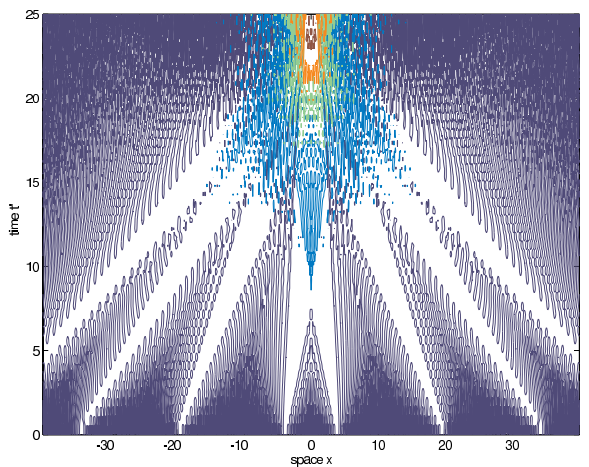

(d)

Fig. 20. Contour plots of the numerical solution of the NLS equation with various choices of frequency jumps: (a) four jumps, with parameters corresponding to the solution shown in Figure 19; (b) six jumps, positioned at $-X_{3}=X_{4}=2,-X_{2}=X_{5}=15,-X_{1}=X_{6}=20$ and all with amplitude $C=2$; (c) six jumps, with the same parameters as before except $-X_{2}=X_{5}=20$ and $-X_{1}=X_{6}=35$; (d) eight jumps, each with amplitude $C=2$ and positioned at $-X_{4}=X_{5}=4$, $-X_{3}=X_{6}=20,-X_{2}=X_{7}=35,-X_{1}=X_{8}=50$.

shows an eight-jump initial condition, with equally sized jumps $C_{j}=2$, generating an overall amplitude $|q|_{\max }=5$. Once more, the jump locations were chosen so that this region emerges near $t^{\prime}=25$. A convenient way to choose the jump locations is to remember that each frequency jump moves with velocity corresponding to the average frequency across the jump.

Finally, we note that the width of the central genus- 0 region can easily be adjusted by changing the temporal separation between the two critical frequency jumps, by virtue of the linear relation between these two quantities, which was discussed in Section 6.

Smooth frequency transitions. The remarks at the end of Section 5 about the general stability of the analytical results with respect to small changes in the initial conditions 


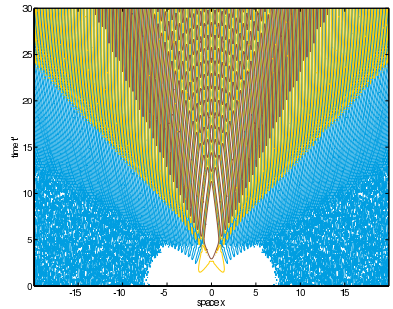

(a)

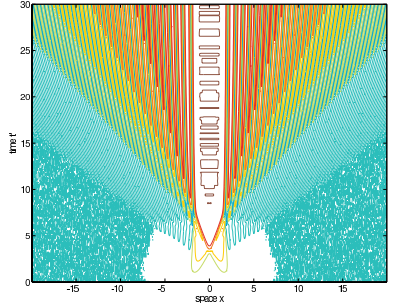

(b)

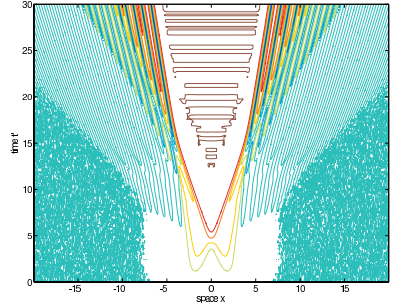

(c)

Fig. 21. Numerical simulations of NLS with smooth frequency transitions, with two frequency jumps located at $X_{2}=-X_{1}=2$ : (a) $C=3$; (b) $C=2$, (c) $C=1$. These figures should be compared to Figure $11 \mathrm{~b}-\mathrm{d}$.

also apply with regard to the discontinuity in the frequency jumps. That is, we expect that the general picture described here will be an approximate description of the pulse behavior even if the frequency transitions were continuous, as would be the case in any experimental setup. To test this prediction, we performed numerical simulations in which, instead of (5.1), the initial frequency was chosen according to

$$
u(x, 0)=\frac{1}{2} \sum_{j=1}^{N} C_{j} \tanh \left(x-X_{j}\right)
$$

which implies $\varphi(x, 0)=(1 / 2) \sum_{j=1}^{N} C_{j} \log \left(\cosh \left(x-X_{j}\right)\right)$. The comparison of the numerical results for discontinuous and smooth frequency transitions shows remarkable agreement between the two cases, with regard to both the bifurcation diagrams and the amplitude of the genus- 0 regions. As an example, in Figure 21 we show numerical simulations of two frequency jumps with the same parameter values as in Figure 11b-d. Note also that the hexagonal pattern near the center $x=0$ in Figure 21a shows a genus2 solution of the NLS equation (see also Figure 11). The evolution of the Riemann invariants corresponding to Figure 21a is given by a smooth version of Figure 8.

Filtering. The high-frequency oscillations that appear on each side of the genus-0 region may be undesirable for practical purposes. These oscillations can effectively be removed by appropriately filtering the pulse at the fiber output, resulting in a smooth, highamplitude short optical pulse. For example, post-processing with a Lorentzian filter with frequency response $F(\omega)=(\sqrt{R} / \pi) /\left(1+R \omega^{2}\right)$, with $R \ll 1$, effectively "wipes out" the genus- 1 and genus- 2 oscillations. A drawback of this method, however, is that, in the case of a narrow pulse, the conversion efficiency (i.e., the amount of energy in the initial CW pulse that is preserved in the final high-amplitude pulse) is rather low after filtering. A higher conversion efficiency can be obtained either by employing an Erbiumdoped fiber amplifier or by increasing the temporal separation between the two central frequency jumps, so as to obtain a wider genus-0 region.

Parameter scaling. As a concluding remark we note that, even though almost all the figures in this work describe solutions produced by initial conditions with amplitude 
$q_{0}=1$, all of these numerical results can easily be related to different values of initial amplitude thanks to the scaling symmetry of the NLS equation. Namely, if $q(x, t) \rightarrow$ $a q(x, t)$, the results in this work still apply upon rescaling $x \rightarrow x^{\prime}=x / a, t \rightarrow$ $t^{\prime}=t / a^{2}$, implying $\rho(x, t) \rightarrow \rho^{\prime}\left(x^{\prime}, t^{\prime}\right)=a^{2} \rho\left(a x^{\prime}, a^{2} t^{\prime}\right)$ and $u(t, x) \rightarrow u^{\prime}\left(x^{\prime}, t^{\prime}\right)=$ $a u\left(a x^{\prime}, a^{2} t^{\prime}\right)$. Importantly, the same scalings can also be used to relate the results to choices of spatial or temporal units different from those discussed in Appendix A.1.

\section{Appendix A.1 Nondimensionalizations and Scalings}

It has been known for more than thirty years that the propagation of a coherent light pulse in optical fibers is governed by the NLS equation, which, in physical units, is [4], [23]

$$
i \frac{\partial E}{\partial z}-\frac{1}{2} k^{\prime \prime} \frac{\partial^{2} E}{\partial t^{2}}+\gamma|E|^{2} E=0,
$$

where $E(t, z)$ is the slowly varying amplitude of the complex envelope of the electric field of the pulse, $t=t_{\text {lab }}-z / c_{g}$ is the retarded time, and $z$ is the propagation distance. Here, $c_{g}=1 / k^{\prime}$ is the group velocity, $k(\omega)$ the propagation constant, $k^{\prime \prime}=d^{2} k / d \omega^{2}$ the dispersion coefficient, and $\gamma$ the nonlinear coefficient. In the following, we will use the typical value $\gamma=3 \mathrm{~W}^{-1} \mathrm{~km}^{-1}$.

Introducing the dimensionless variables $q=E / \sqrt{P_{*}}, T=t / t_{*}$, and $Z=z / z_{\mathrm{nl}}$, where $P_{*}$ and $t_{*}$ are some characteristic power and temporal duration, and where $z_{*}=z_{\mathrm{nl}}=$ $1 /\left(\gamma P_{*}\right)$ is the nonlinear length (the characteristic distance over which nonlinear effects take place), (A.1) becomes

$$
i \frac{\partial q}{\partial Z}-\frac{1}{2} \bar{d} \frac{\partial^{2} q}{\partial T^{2}}+|q|^{2} q=0
$$

The dimensionless dispersion coefficient $\bar{d}=k^{\prime \prime} / k_{*}^{\prime \prime}=z_{\mathrm{nl}} / z_{\text {disp }}$ (with $k_{*}^{\prime \prime}=t_{*}^{2} / z_{\mathrm{nl}}$ ) quantifies the relative importance of nonlinear and dispersive effects in (A.1). In this work we are interested in particular in situations where $0<\bar{d} \ll 1$. This can be achieved in two different ways: constant dispersion or dispersion management. Note that the dimensionless dispersion coefficient $\bar{d}$ in (A.2) is obtained equivalently as $\bar{d}=k^{\prime \prime} / k_{*}$ or as $\bar{d}=D / D_{*}$, with $D=-\left(2 \pi c / \lambda^{2}\right) k^{\prime \prime}$, with $D_{*}=-\left(2 \pi c / \lambda^{2}\right) k_{*}^{\prime \prime}$, and where $D$ is the dispersion coefficient in $\mathrm{ps} /(\mathrm{nm} \cdot \mathrm{km})$. Note $2 \pi c / \lambda^{2}=0.78(\mathrm{~nm} \cdot \mathrm{ps})^{-1}$ at $\lambda=1.55 \mu \mathrm{m}$.

Typical units. We first consider the case of fibers with constant dispersion. A unit power $P_{*}=2 \mathrm{~W}$ (which can be obtained using commercially available high-power Erbium-Doped-Fiber-Amplifier lasers) implies $z_{\mathrm{nl}}=0.16 \mathrm{~km}$. A characteristic time unit of $t_{*}=50 \mathrm{fs}$ (which is appropriate in order to describe short optical pulses) yields $k_{*}^{\prime \prime}=1.5 \mathrm{ps}^{2} / \mathrm{km}$. Then the dimensionless dispersion coefficient $\bar{d}$ in (A.2) is $\bar{d}=$ $D / D_{*}$, with $D_{*}=-1.2 \mathrm{ps} /(\mathrm{nm} \cdot \mathrm{km})$. A value of $\bar{d}=0.1$ (such as the one used in all the simulations presented in this work) can then be obtained by employing a fiber with dispersion coefficient $D=-0.12 \mathrm{ps} /(\mathrm{nm} \cdot \mathrm{km})$. Alternatively, the same effects can 
be observed for lower-amplitude, longer pulses over longer propagation distances: for example, with a unit power $P_{*}=20 \mathrm{~mW}$, and a unit time $t_{*}=0.5 \mathrm{ps}$, the unit distance would be $z_{*}=16 \mathrm{~km}$.

Fibers with a larger nonlinear coefficient (such as a dispersion-shifted fiber or a photonic-crystal fiber) would have a correspondingly smaller nonlinear length $z_{\mathrm{nl}}$, which would make it possible to observe the same nonlinear effects over shorter distances. Also, for comparable values of $t_{*}$ and $P_{*}$, a higher nonlinearity would result in a larger value of $k_{*}^{\prime \prime}$, which means that fibers with correspondingly larger dispersion coefficients may be used. Alternatively, a higher nonlinearity would make it possible to achieve the same value of $k_{*}^{\prime \prime}$ (and hence $\bar{d}$ ) with shorter temporal scales $t_{*}$ and/or with smaller peak powers $P_{*}$.

It should be noted that (A.1) neglects the effect of fiber loss, and therefore the peak powers listed above should be taken as the average of the pulse powers over the whole transmission span. This is not a serious issue if the total propagation distance is much shorter than the characteristic distances for fiber absorption, as with the sample parameters chosen earlier (for standard telecommunication fibers, a power loss coefficient of $0.2 \mathrm{~dB} / \mathrm{km}$ is typical), or if the effects of damping can be minimized by placing one or more (Erbium-doped and/or Raman) fiber amplifiers inside the transmission span, since it is well known that the average dynamics in systems with loss and periodic amplification are still governed by the NLS (1.1) with constant coefficients [21]. We should also note that quasi-lossless propagation of optical pulses over several hundred kilometers has recently been achieved by making use of two bidirectional Raman pumps plus fiber Bragg gratings [13], [5]. Finally, note that (A.1) also neglects higher-order dispersion. As shown in [27], third-order dispersion can affect pulse behavior. In order for the results described in this work to apply, therefore, it is necessary that the fiber dispersion be approximately constant throughout the bandwidth of the pulse, which means that third-order dispersion should be small.

Dispersion management. If realizing small values of $\bar{d}$ in a stable way should require an unpractical level of control over fiber dispersion, the problem might be circumvented by making use of dispersion management. With dispersion management, a very small value of $\epsilon$ can be achieved as an average between the opposite dispersion coefficients of fibers with alternating signs of dispersion, even if the local values of dispersion are not individually small.

The propagation of optical pulses in systems with dispersion management is still described by the NLS eq. (A.2); however, the dimensionless dispersion coefficient $\bar{d}$ in (A.2) is replaced by a periodic function $d(z)$. The specific choice of $d(z)$ is called a dispersion map. The relative effect of the periodic dispersion variations can be quantified by the reduced map strength parameter $s$, which is defined as [1] $4 s=\langle|d(\cdot)-\bar{d}|\rangle z_{a}$, where now $\bar{d}=\langle d(\cdot)\rangle$ and the average is taken over the period of the dispersion map, $z_{a}$. As long as the strength $s$ of the dispersion map is small, it is possible to average over the rapid variations of the pulse profile originating in the NLS equation with periodic coefficients. Indeed, it is well known that, just as with loss/amplification, the NLS eq. (A.2) describes the evolution of the leading-order portion of the pulse envelope [22] in a system with a moderate amount of dispersion management. That is, 
the leading order part of the pulse again satisfies the NLS equation (A.2), except that the parameter $\bar{d}$ now represents the average dispersion. Therefore, the use of dispersion management might be desirable because it allows one to more easily obtain small values of effective dispersion than for a system with constant dispersion. Because on average the system is still governed by the NLS equation, the results presented in this work will be preserved as long as the dispersion map strength $s$ is small. If the map strength $s$ becomes large, however, the leading order dynamics are described not by the NLS, but rather by a nonlocal evolution equation of NLS-type [1], [17]. Determining the behavior of the solutions with small $\bar{d}$ for this type of system is a highly nontrivial task, which is beyond the scope of this work.

\section{Appendix A.2 Genus-1 Solutions of NLS and Whitham's Averaging Method}

Here we briefly review the construction of genus- 1 solutions of the NLS equation and the derivation of the genus-1 NLS-Whitham equations, with the aim of clarifying the connection between the Riemann invariants and the spectrum of the Lax operator. For a detailed construction of higher-genus solutions involving theta functions, see [6], [18].

In order to construct traveling wave solutions of the NLS equation (1.1), it is convenient to introduce the fast space and time scales $x^{\prime}=x / \epsilon$ and $t^{\prime}=t / \epsilon$, and write (1.1) as $i q_{t^{\prime}}-\frac{1}{2} q_{x^{\prime} x^{\prime}}+|q|^{2} q=0$. We then look for solutions of the form

$$
q\left(x^{\prime}, t^{\prime}\right)=e^{i\left[c x^{\prime}+\frac{1}{2}\left(c^{2}+\Omega^{2}\right) t^{\prime}\right]} f\left(x^{\prime}+c t^{\prime}\right),
$$

with $c, \Omega \in \mathbb{R}$ and $f(\cdot)$ a real function of its argument. Substituting (A.3) into the NLS equation and integrating once, one obtains

$$
(d f / d x)^{2}=f^{4}-\sigma_{1} f^{3}+\sigma_{2} f^{2}-\sigma_{3} f+\sigma_{4}=: \mu(f),
$$

where $\sigma_{1}=\sigma_{3}=0, \sigma_{2}=-\Omega^{2}, \sigma_{4}=C$, and where $C$ is the integration constant. It is convenient to represent the right-hand side of (A.4) as

$$
\mu(f)=\left(f-r_{1}\right)\left(f-r_{2}\right)\left(f-r_{3}\right)\left(f-r_{4}\right),
$$

where the roots $r_{1}, \ldots, r_{4}$ are related to the coefficients in (A.4) by

$$
\begin{gathered}
\sigma_{1}=r_{1}+r_{2}+r_{3}+r_{4}, \quad \sigma_{2}=r_{1} r_{2}+r_{1} r_{3}+r_{1} r_{4}+r_{2} r_{3}+r_{2} r_{4}+r_{3} r_{4}, \text { (A.6a) } \\
\sigma_{3}=r_{1} r_{2} r_{3}+r_{1} r_{2} r_{4}+r_{1} r_{3} r_{4}+r_{2} r_{3} r_{4}, \quad \sigma_{4}=r_{1} r_{2} r_{3} r_{4} .
\end{gathered}
$$

Integrating (A.4) formally one has $\int d f / \sqrt{\mu(f)}= \pm\left(x^{\prime}-x_{0}^{\prime}\right)$. The integral then defines the solution of NLS in terms of elliptic functions. Note, however, that real solutions are confined to those values of $f$ such that $\mu(f)>0$. It is then clear that the values of the four roots $r_{1}, \ldots, r_{4}$ (which will be the Riemann invariants when performing the Whitham averaging described in the next subsection) determine the edges of the forbidden regions, which are the values of $f$ for which $\mu(f)<0$. Several situations can occur depending on the values of the parameters in (A.4). It is relatively easy, however, to see that bounded 
solutions only exist when all four roots of $\mu(f)$ are real. In this case the solution of (A.4) is given by the elliptic sine [9]: $f\left(x^{\prime}\right)=k \operatorname{sn}\left(x^{\prime}, k\right)$, where the elliptic modulus $k \in[0,1]$ is given by $k^{2}=\Omega^{2}-1=C$. (The elliptic parameter [3] is $m=k^{2}$.) If we label the roots of $\mu(f)$ as $r_{1}<r_{2}<r_{3}<r_{4}$, it is $r_{4}=-r_{1}=1$ and $r_{3}=-r_{2}=k$. The value of $f$ oscillates between $r_{2}$ and $r_{3}$, and the spatial period of the solution is given by $4 K(k)$, where $K(k)$ is the elliptic integral of the first kind [3]. In the limiting case $r_{4}=r_{3}=-r_{1}=-r_{2}$, one has $k=1$, the period of the solution tends to infinity, and one obtains the dark soliton solutions of the NLS equation: $f\left(x^{\prime}\right)=\tanh x^{\prime}$. Note that the solution of the NLS equation is specified in terms of the four roots $r_{1}, \ldots, r_{4}$. For a more detailed discussion of single-phase solutions of the NLS equation in the focusing as well as defocusing regimes, as well as their stability, see [10], [26].

It is important to note that, for any finite value of the small parameter $\epsilon$, the presence of $\epsilon$ in (1.1) can be taken into account by simply replacing $(x, t)$ with the fast variables $\left(x^{\prime}=x / \epsilon, t^{\prime}=t / \epsilon\right)$. This phenomenon is the origin of the high-frequency oscillations in the solution of NLS with small dispersion coefficient, discussed throughout this work. The nontrivial issue, however, is to understand the qualitative behavior of the solution in the weak dispersion limit, as formulated in Section 1.

Let us then briefly outline how to obtain the genus-1 NLS-Whitham equations. It is well known that the NLS equation admits an infinite number of conservation laws:

$$
\frac{\partial F_{n}}{\partial t^{\prime}}=\frac{\partial G_{n}}{\partial x^{\prime}}
$$

$n=1, \ldots, \infty$. For example, the first four conserved densities are

$$
\begin{gathered}
F_{1}=|q|^{2} \\
F_{2}=i\left(q^{*} q_{x^{\prime}}-q q_{x^{\prime}}^{*}\right), \\
F_{3}=\left|q_{x^{\prime}}\right|^{2}+|q|^{4}, \\
F_{4}=i\left(q^{*} q_{x^{\prime} x^{\prime} x^{\prime}}-q q_{x^{\prime} x^{\prime} x^{\prime}}^{*}\right)-3 i|q|^{2}\left(q^{*} q_{x^{\prime}}-q q_{x^{\prime}}^{*}\right) .
\end{gathered}
$$

Then we consider small modulations of the genus-1 solution given by the elliptic function, in the sense that the deformation due to the modulation is of order $\epsilon$ over one period of the wave. Following the standard perturbation method of multiple scales, we introduce the slow time and space scales $x=\epsilon x^{\prime}$ and $t=\epsilon t^{\prime}$. We now have two sets of scales, consisting of a set of fast scales for the oscillations and a set of slow scales for the modulations. With those variables, we have

$$
\frac{\partial}{\partial x^{\prime}} \rightarrow \frac{\partial}{\partial x^{\prime}}+\epsilon \frac{\partial}{\partial x}, \quad \frac{\partial}{\partial t^{\prime}} \rightarrow \frac{\partial}{\partial t^{\prime}}+\epsilon \frac{\partial}{\partial t} .
$$

Then, expanding the conserved quantities as $F_{n}=F_{n}^{0}+\epsilon F_{n}^{1}+\cdots$ and $G_{n}=G_{n}^{0}+$ $\epsilon G_{n}^{1}+\cdots$ and taking the average of the conservation laws (A.7) over the fast scale $x^{\prime}$, at order $\epsilon$ we obtain the NLS-Whitham equations in conservation form:

$$
\frac{\partial\left\langle F_{n}^{0}\right\rangle}{\partial t}=\frac{\partial\left\langle G_{n}^{0}\right\rangle}{\partial x}
$$


Here $\left\langle F_{n}^{0}\right\rangle$ and $\left\langle G_{n}^{0}\right\rangle$ are the average of the leading-order conserved densities and fluxes associated with the solution of the NLS equation, and the average of a periodic function $f\left(x^{\prime}, t^{\prime}\right)=\hat{f}(\theta)$ is given by

$$
\langle f\rangle=\frac{1}{L} \int_{-L / 2}^{L / 2} f\left(x^{\prime}, t^{\prime}\right) d x^{\prime}=\int_{0}^{1} \hat{f}(\theta) d \theta,
$$

where $L$ is the spatial period and $\theta=\left(x^{\prime}+c t^{\prime}\right) / L$. For the genus- 1 solutions described above, $L=4 K(k)$. Note that the averaged quantities $\left\langle F_{n}^{0}\right\rangle$ and $\left\langle G_{n}^{0}\right\rangle$ are functions of the spectral parameters $r_{1}, \ldots, r_{4}$, Thus the modulations are expressed in terms of the slow motion of the spectral parameters $r_{1}, \ldots, r_{4}$. This also means that we need the first four equations from (A.9). By computing the averages, one finds that the variables $r_{1}, \ldots, r_{4}$ are precisely the Riemann invariants for the system. Then, by writing (A.9) in diagonal form, one finally obtains the genus-1 NLS-Whitham equations (2.3) with $g=1$. (That (A.9) are diagonalizabile is not obvious, but always true for all values of genus; see Section A.3 for further details.)

\section{Appendix A.3 The NLS-Whitham Equations}

Here we briefly review some well-known results for the NLS-Whitham equations. For more details on the finite-genus solutions of the NLS equation, the averaging process, and the NLS-Whitham equations, we refer the reader to [6], [27], and references therein.

The genus- $g$ NLS-Whitham equations can be obtained by extending the approach described in Section A. 2 to hyperelliptic solutions of NLS and $2 g+2$ conservation laws [15]. It is more convenient, however, to take advantage of the integrable structure of the NLS equation, as described in [27]. Recall that genus-g solutions of NLS are associated with the Riemann surface $R: w^{2}=\mu_{g}(z)$, where $\mu_{g}(z)=\prod_{k=1}^{2 g+2}\left(z-r_{k}\right)$ (cf. (2.2)). It was shown in [14], [15], [29], [31] that the NLS-Whitham equations (A.9) can be elegantly written as

$$
\frac{\partial \omega_{1}}{\partial t}=\frac{\partial \omega_{2}}{\partial x},
$$

where $\omega_{1}$ and $\omega_{2}$ are the meromorphic (Abelian) differentials of the second kind associated with the Riemann surface $R$ :

$$
\begin{aligned}
\omega_{1} & =\frac{1}{2}\left[1+\frac{z^{g+1}-\frac{1}{2} \sigma_{1} z^{g}+\alpha_{1} z^{g-1}+\cdots+\alpha_{g-1} z+\alpha_{g}}{\sqrt{\mu_{g}(z)}}\right] d z \\
& =(1+O(1 / z)) d z, \\
\omega_{2} & =\frac{1}{2}\left[z+\frac{z^{g+2}-\frac{1}{2} \sigma_{1} z^{g+1}+\frac{1}{2}\left(\sigma_{2}-\frac{1}{4} \sigma_{1}^{2}\right) z^{g}+\gamma_{1} z^{g-1}+\cdots+\gamma_{g-1} z+\gamma_{g}}{\sqrt{\mu_{g}(z)}}\right] d z \\
& =(z+O(1 / z)) d z,
\end{aligned}
$$

where $\sigma_{1}, \ldots, \sigma_{3}$ are the generalization of (A.6),

$$
\sigma_{1}=\sum_{1 \leq j \leq 2 g+2} r_{j}, \quad \sigma_{2}=\sum_{1 \leq j<k \leq 2 g+2} r_{j} r_{k}, \quad \sigma_{3}=\sum_{1 \leq i<j<k \leq 2 g+2} r_{i} r_{j} r_{k}
$$


( $\sigma_{3}$ will appear later on), and where the coefficients $\alpha_{j}$ and $\gamma_{j}$ are given by the solution of two $g \times g$ linear systems of equations

$$
\begin{array}{r}
I_{j}^{g+1}-\frac{1}{2} \sigma_{1} I_{j}^{g}+\alpha_{1} I_{j}^{g-1}+\cdots+\alpha_{g-1} I_{j}^{1}+\alpha_{g} I_{j}^{0}=0, \\
I_{j}^{g+2}-\frac{1}{2} \sigma_{1} I_{j}^{g+1}+\frac{1}{2}\left(\sigma_{2}-\frac{1}{4} \sigma_{1}^{2}\right) I_{j}^{g}+\gamma_{1} I_{j}^{g-1}+\cdots+\gamma_{g-1} I_{j}^{1}+\gamma_{g} I_{j}^{0}=0,
\end{array}
$$

$j=1, \ldots, g$, with the real-valued functions $I_{j}^{k}$ given by

$$
I_{j}^{k}=\frac{1}{2} \int_{r_{2 j+1}}^{r_{2 j+2}} \frac{z^{k}}{\sqrt{-\mu_{g}(z)}} d z
$$

Equations (A.15) express the requirement that the A-periods of $\omega_{1}, \omega_{2}$ be zero: that is, $\oint_{a_{j}} \omega_{1}=\oint_{a_{j}} \omega_{2}=0$, where the $a_{j}$ are the A-cycles on the Riemann surface $R$.

Evaluating the residue of $\omega_{1,2}$ at $z=r_{k}$ in (A.11), one obtains the genus- $g$ NLSWhitham equations in Riemann invariant form:

$$
\frac{\partial r_{k}}{\partial t^{\prime}}=s_{k}\left(r_{1}, \ldots, r_{2 g+2}\right) \frac{\partial r_{k}}{\partial x},
$$

$k=1, \ldots, 2 g+2$. The characteristic speeds are given by $s_{k}\left(r_{1}, \ldots, r_{2 g+2}\right)=s\left(r_{k}\right)$, with

$$
s(r)=\frac{r^{g+2}-\frac{1}{2} \sigma_{1} r^{g+1}+\frac{1}{2}\left(\sigma_{2}-\frac{1}{4} \sigma_{1}^{2}\right) r^{g}+\gamma_{1} r^{g-1}+\cdots+\gamma_{g-1} r+\gamma_{g}}{r^{g+1}-\frac{1}{2} \sigma_{1} r^{g}+\alpha_{1} r^{g-1}+\cdots+\alpha_{g-1} r+\alpha_{g}} .
$$

Since the NLS-Whitham equations (A.16) describe the evolution of the Riemann invariants $r_{1}, \ldots, r_{2 g+2}$ with respect to the slow time and space variables, and since the Riemann invariants are also the branch points of the Riemann surface corresponding to finite-genus solutions of the NLS equation, it follows that the NLS-Whitham equations describe the slow modulation of finite-genus solutions of the NLS equation.

Note that the Abelian differentials in (A.12) can also be expanded in terms of the conservation laws of the NLS equation:

$$
\omega_{1} \sim\left(1+\sum_{n=1}^{\infty} \frac{n\left\langle F_{n}^{0}\right\rangle}{z^{n+1}}\right) d z, \quad \omega_{2} \sim\left(z+\sum_{n=1}^{\infty} \frac{n\left\langle G_{n}^{0}\right\rangle}{z^{n+1}}\right) d z,
$$

where $\left\langle F_{n}^{0}\right\rangle$ and $\left\langle G_{n}^{0}\right\rangle$ are the conserved densities and fluxes of the NLS equation, averaged over the fast phases. The average of a quasi-periodic function of $g$ phases $f\left(x^{\prime}, t^{\prime}\right)=$ $\hat{f}\left(\theta_{1}, \ldots, \theta_{g}\right)$, with $\theta_{k}=\left(x^{\prime}+c_{k} t^{\prime}\right) / L_{k}$ and $L_{k}$ being the period for the phase $\theta_{k}$, is given by

$$
\langle f\rangle=\lim _{L \rightarrow \infty} \frac{1}{L} \int_{-L / 2}^{L / 2} f\left(x^{\prime}, t^{\prime}\right) d x^{\prime}=\int_{0}^{1} \cdots \int_{0}^{1} \hat{f}\left(\theta_{1}, \ldots, \theta_{g}\right) d \theta_{1} \cdots d \theta_{g},
$$

where we have used the ergodic assumption, i.e., that the $g$ phases produce a dense orbit on the Jacobian variety of the Riemann surface [14]. In this framework (A.11) then yield 
(A.9) for $n=1, \ldots, 2 g+2$. For example, the first few densities and fluxes are

$$
\begin{aligned}
& \left\langle F_{1}^{0}\right\rangle=\langle\rho\rangle=-\frac{1}{4} \sigma_{2}+\frac{1}{16} \sigma_{1}^{2}+\frac{1}{2} \alpha_{1}, \\
& \left\langle F_{2}^{0}\right\rangle=\langle\rho u\rangle=-\frac{1}{4} \sigma_{3}-\frac{1}{8} \sigma_{1} \sigma_{2}+\frac{1}{32} \sigma_{1}^{3}+\frac{1}{8} \sigma_{1} \alpha_{1}+\frac{1}{4} \alpha_{2}, \\
& \left\langle G_{1}^{0}\right\rangle=\left\langle F_{2}^{0}\right\rangle=-\frac{1}{4} \sigma_{3}-\frac{1}{8} \sigma_{1} \sigma_{2}+\frac{1}{32} \sigma_{1}^{3}+\frac{1}{2} \gamma_{1}, \\
& \left\langle G_{2}^{0}\right\rangle=\left\langle\rho u^{2}+\frac{1}{2} \rho^{2}\right\rangle=-\frac{1}{8} \sigma_{4}+\frac{1}{8} \sigma_{1} \sigma_{3}+\frac{1}{32} \sigma_{2}^{2}-\frac{5}{64} \sigma_{1}^{2} \sigma_{2}+\frac{9}{512} \sigma_{1}^{4}+\frac{1}{8} \sigma_{1} \gamma_{1}+\frac{1}{4} \gamma_{2},
\end{aligned}
$$

and (1.5) is obtained from (A.9) when $g=0$.

Upon evaluating the integrals in (A.15) and taking the appropriate limits as $\epsilon \rightarrow 0^{+}$, (A.17) can be used to calculate the value of all the speeds listed in Sections 3 and 4, as we show in the next subsection. It should be noted that the general solution of the NLS-Whitham equations (A.16) can be written in terms of the Hodograph transformation

$$
x-s_{k}\left(r_{1}, \ldots, r_{2 g+2}\right) t=w_{k}\left(r_{1}, \ldots, r_{2 g+2}\right),
$$

$k=1, \ldots, 2 g+2$, where the functions $w_{k}$ are expressed in terms of the initial data via hyperelliptic integrals. When inverting (A.20) to find the solution $q(x, t)$, the locations where the Jacobian of the transformation vanishes determine the boundary between regions of different genus [33], [34]. This information was used in [33] to determine the location between regions of genus- 0 and genus- 1 in some specific situations, and the method was used in [19] to determine the generic evolution of an arbitrary initial datum for the Korteweg-de Vries equation. It should be noted, however, that the method cannot be easily applied for the situations considered in the present work, since the transformation $x \rightarrow q(x, 0)$ is not invertible in the case of piecewise-constant initial data, which are the ones of interest here.

\section{Appendix A.4 Calculation of Some Characteristic Speeds}

We now briefly describe some calculations regarding the characteristic speeds of the regularized Riemann invariants for the NLS-Whitham equations. The results of these calculations and similar others were summarized in Sections 3 and 4. Since the calculations are rather tedious, however, we will limit ourselves to presenting two examples. For more details on similar types of calculations, we also refer the reader to [8], [27]. When performing these calculations, it is useful to note that the characteristic speed in (A.17) is the ratio of two meromorphic differentials, which becomes the ratio of two polynomials (the denominator of the differentials). In the case of step initial data, some common factors then arise, and the resulting cancellations produce a finite result.

Genus-1 calculations: Equations (3.1) and (4.5). Our first aim is to calculate $s_{3}^{-}$in case (i) of Section 3, as shown in Figure 4a. This is done by evaluating $s_{3}\left(r_{1}, \ldots, r_{4}\right)=$ $s\left(r_{3}\right)$, with $s(r)$ given by (A.17), with $r_{4}=-r_{1}=u_{0}+2 q_{0}, r_{2}=-u_{0}+2 q_{0}$, and $r_{3}=r_{4}-\epsilon$, and then taking the limit $\epsilon \rightarrow 0^{+}$. In general, (A.15) yield the coefficients $\alpha_{1}$ and $\gamma_{1}$ for the $g=1$ case as

$$
\alpha_{1}=-\frac{I_{1}^{2}-\frac{1}{2} \sigma_{1} I_{1}^{1}}{I_{1}^{0}}, \quad \gamma_{1}=-\frac{I_{1}^{3}-\frac{1}{2} \sigma_{1} I_{1}^{2}+\frac{1}{2}\left(\sigma_{2}-\frac{1}{4} \sigma_{1}^{2}\right) I_{1}^{1}}{I_{1}^{0}} .
$$


In our case, (A.13) also give $\sigma_{1}=r_{2}+r_{4}=4 q_{0}+\epsilon$ and $\sigma_{2}=r_{2} r_{4}-r_{3}^{2}=-2 u_{0}\left(u_{0}+\right.$ $\left.2 q_{0}\right)+\epsilon\left(u_{0}-2 q_{0}\right)$. In general, upon making the substitution $z=r_{3}+\Delta \sin ^{2} \theta$ with $\Delta=r_{4}-r_{3}$, the integrals $I_{1}^{0}, \ldots, I_{1}^{3}$ take the convenient form:

$$
I_{1}^{k}=\int_{0}^{\pi / 2} \frac{\left(r_{3}+\Delta \sin ^{2} \theta\right)^{k}}{\sqrt{\left(r_{3}-r_{1}+\Delta \sin ^{2} \theta\right)\left(r_{3}-r_{2}-\Delta \sin ^{2} \theta\right)}} d \theta .
$$

In our case, expanding the power in the numerator and substituting the explicit value of the invariants, the integrals can also be written as

$$
I_{1}^{k}=\sum_{m=0}^{k}\left(\begin{array}{l}
k \\
m
\end{array}\right)\left(u_{0}+2 q_{0}\right)^{k-m} \epsilon^{m} \int_{0}^{\pi / 2} \frac{\sin ^{2 m} \theta}{\sqrt{\left(2\left(u_{0}+2 q_{0}\right)-\epsilon \cos ^{2} \theta\right)\left(2 u_{0}-\epsilon \cos ^{2} \theta\right)}} d \theta .
$$

Then, evaluating the integrals in (A.23), substituting the results and (A.21) into (A.17) and taking the limit $\epsilon \rightarrow 0^{+}$, one obtains the desired result, namely (3.1). Note that, even though $\lim _{\epsilon \rightarrow 0^{+}} I_{1}^{0}=\infty$, the final result for the characteristic speed is finite.

We should emphasize that, by rescaling $u_{0} \rightarrow 2 u_{0}$, the above calculation also provides the speed $s_{\text {outer }}$ in (4.5) of Section 4. More precisely, upon neglecting degenerate branches in Figure $6 \mathrm{a}-\mathrm{c}$, the values of the Riemann invariants that determine $s_{3}^{-}$in Figure $4 \mathrm{a}$ are exactly those of the Riemann invariants that determine $s_{5}^{-}$in case (i) (cf. Figure 6a) and $s_{9}^{-}$in cases (ii) and (iii) (cf. Figure 6b,c) of Section 4 upon replacing $u_{0} \rightarrow 2 u_{0}$.

Degenerate genus-2 calculations: Equations (3.2) and (4.8). We now want to calculate $s_{5}^{+}$in case (ii) of Section 3, as shown in Figure 4b. This is done by evaluating $s_{5}\left(r_{1}, \ldots, r_{6}\right)=s\left(r_{5}\right)$, with $s(r)$ still given by (A.17) with $g=2$ and with $r_{6}=-r_{1}=u_{0}+2 q_{0}, r_{4}=-r_{3}=-u_{0}+2 q_{0}, r_{5}=-r_{2}=r_{4}+\epsilon$, and then again taking the limit $\epsilon \rightarrow 0^{+}$. In general, (A.15) yield the coefficients $\alpha_{1} \alpha_{2}, \gamma_{1}$, and $\gamma_{2}$ for the $g=2$ case as

$$
\begin{aligned}
\alpha_{j+1} & =(-1)^{j+1} \frac{1}{[1,0]}\left([3, j]-\frac{1}{2} \sigma_{1}[2, j]\right), \\
\gamma_{j+1} & =(-1)^{j+1} \frac{1}{[1,0]}\left([4, j]-\frac{1}{2} \sigma_{1}[3, j]+\frac{1}{2}\left(\sigma_{2}-\frac{1}{4} \sigma_{1}^{2}\right)[2, j]\right),
\end{aligned}
$$

$j=0,1$, where

$$
[i, j]=\operatorname{det}\left(\begin{array}{cc}
I_{1}^{i} & I_{1}^{j} \\
I_{2}^{i} & I_{2}^{j}
\end{array}\right) .
$$

In our case, thanks to the symmetry of the initial datum for the invariants, (A.13) give $\sigma_{1}=0$ and $\sigma_{2}=-r_{4}^{2}-r_{5}^{2}-r_{6}^{2}=\left(-3 u_{0}^{2}+4 u_{0} q_{0}-12 q_{0}^{2}\right)+2\left(u_{0}-2 q_{0}\right) \epsilon-\epsilon^{2}$. Upon making the substitutions $z=r_{3}+\left(r_{4}-r_{3}\right) \sin ^{2} \theta$ on $I_{1}^{k}$ and $z=r_{5}+\left(r_{6}-r_{5}\right) \sin ^{2} \theta$ on $I_{2}^{k}$ and using the reflective symmetry of the initial datum, the integrals $I_{1,2}^{0}, \ldots, I_{1,2}^{4}$ take the form

$$
I_{1}^{k}=(-1)^{k} r_{4}^{k} \int_{0}^{\pi / 2} \frac{\cos ^{2 k} 2 \theta}{\sqrt{\left(r_{5}^{2}-r_{4}^{2} \cos ^{2} 2 \theta\right)\left(r_{6}^{2}-r_{4}^{2} \cos ^{2} 2 \theta\right)}} d \theta,
$$




$$
I_{2}^{k}=\int_{0}^{\pi / 2} \frac{\left(r_{5}+\Delta \sin ^{2} \theta\right)^{k}}{\sqrt{\left(r_{5}-r_{4}+\Delta \sin ^{2} \theta\right)\left(r_{4}+r_{5}+\Delta \sin ^{2} \theta\right)\left(2 r_{5}+\Delta \sin ^{2} \theta\right)\left(r_{5}+r_{6}+\Delta \sin ^{2} \theta\right)}} d \theta,
$$

where $\Delta=r_{6}-r_{5}$. Then, evaluating the above integrals, substituting (A.27) and (A.25) into (A.17) and taking the limit $\epsilon \rightarrow 0^{+}$, one obtains the desired result, namely (3.2). Again, note that even though some of the integrals are divergent in the limit $\epsilon \rightarrow 0^{+}$, the result for the characteristic speeds is finite.

Again, we emphasize that, by rescaling $q_{0} \rightarrow q_{0}+\frac{1}{2} u_{0}$ and $u_{0} \rightarrow 2 u_{0}$, the above calculation also provides both of the speeds $s_{\text {inner }}^{(1)}$ and $s_{\text {inner }}^{(2)}$ in (4.7) of Section 4. More precisely, it is easy to see that, upon neglecting degenerate branches in Figure 6b-d, the values of the Riemann invariants that determine $s_{5}^{+}$in Figure $4 \mathrm{~b}$ are the same as those that determine $s_{9}^{+}$in Figure 6b-c $q_{0} \rightarrow q_{0}+\frac{1}{2} u_{0}$. Similarly, upon neglecting degenerate branches Figure $6 \mathrm{~b}-\mathrm{d}$, one sees that the values that determine $s_{5}^{+}$in Figure $4 \mathrm{~b}$ also coincide with those that determine $s_{7}^{+}$in Figure $6 \mathrm{~b}$ and $s_{5}^{+}$in Figure $6 \mathrm{c}$ upon $u_{0} \rightarrow 2 u_{0}$.

\section{Acknowledgments}

We thank W. L. Kath and M. Fiorentino for providing technical specifications regarding appropriate experimental devices, F.-R. Tian for useful discussions on the Whitham equations, and M. Hoefer for his careful reading of the text. This work was partially supported by the National Science Foundation under grant numbers DMS-0404931 and DMS-0506101.

\section{References}

[1] M. J. Ablowitz and G. Biondini, "Multiple scale dynamics in communication systems with strong dispersion management," Opt. Lett. 23, 1668-1670 (1998).

[2] M. J. Ablowitz and H. Segur, Solitons and the Inverse Scattering Transform (Society for Industrial and Applied Mathematics, Philadelphia, 1981).

[3] M. Abramowitz and I. A. Stegun, Handbook of Mathematical Functions (Dover, New York, 1972).

[4] G. P. Agrawal, Nonlinear Fiber Optics (Academic Press, San Diego, 1995).

[5] J. D. Ania-Castañón, T. J. Ellingham, R. Ibbotson, X. Chen, L. Zhang, and S. K. Turitsyn, "Ultralong Raman fiber lasers as virtually lossless optical media," Phys. Rev. Lett. 96, 023902:1-4 (2006).

[6] E. D. Belokolos, A. I. Bobenko, V. Z. Enolskii, A. R. Its, and V. B. Matveev, Algebrogeometric Approach to Nonlinear Integrable Equations (Springer, New York, 1994).

[7] A. M. Bloch and Y. Kodama, "The Whitham equation and shocks in the Toda lattice," 1-19, in Singular Limits of Dispersive Waves, N. M. Ercolani, I. R. Gabitov, C. D. Levermore, and D. Serre, eds. (Plenum, New York, 1994).

[8] A. M. Bloch and Y. Kodama, "Dispersive regularization of the Whitham equation for the Toda lattice," SIAM J. Appl. Math. 52, 909-928 (1992).

[9] P. F. Byrd and M. D. Friedman, Handbook of Elliptic Integrals for Scientists and Engineers (Springer-Verlag, Berlin, 1954).

[10] J. D. Carter and H. Segur, "Instabilities in the two-dimensional cubic nonlinear Schrödinger equation," Phys. Rev. E 68, 045601 (2003). 
[11] G. A. El and A. L. Krylov, "General solution of the Cauchy problem for the defocusing NLS equation in the Whitham limit," Phys. Lett. A 203, 77-82 (1995).

[12] G. A. El, V. V. Geogjaev, A. V. Gurevich and A. L. Krylov, "Decay of an initial discontinuity in the defocusing NLS hierarchy," Phys. D 87, 186-192 (1995).

[13] T. J. Ellingham, J. D. Ania-Castañón, R. Ibbotson, X. Chen, L. Zhang, and S. K. Turitsyn, "Quasi-lossless optical links for broadband transmission and data processing," Photon. Technol. Lett. 18, 268-270 (2006).

[14] H. Flaschka, M. G. Forest and D. W. McLaughlin, "Multiphase averaging and the inverse spectral solution of the Korteweg-de Vries equation," Commun. Pure and Appl. Math. 33, 739-784 (1980).

[15] M. G. Forest and J.-E. Lee, "Geometry and modulation theory for periodic nonlinear Schrödinger equation," in Oscillation Theory, Computation and Methods of Compensated Compactness, IMA vol. Appl. Math. 2, C. Dafermos et al., eds. (Springer, New York, 1986).

[16] M. G. Forest and K. T.-R. McLaughlin, "Onset of oscillations in nonsoliton pulses in nonlinear dispersive fibers,” J. Nonlin. Sci. 7, 43-62 (1998).

[17] I. R. Gabitov and S. K. Turitsyn, "Averaged pulse dynamics in a cascaded transmission system with passive dispersion compensation," Opt. Lett. 21, 327-329 (1996).

[18] F. Gesztesy and H. Holden, Soliton Equations and Their Algebro-Geometric Solutions (Cambridge University Press, Cambridge, 2003).

[19] T. Grava and F.-R. Tian, "The generation, propagation, and extinction of multiphases in the KdV zero-dispersion limit," Commun. Pure Appl. Math. 55, 1569-1639 (2002).

[20] H. Hammack, N. Scheffner, and H. Segur, "Two-dimensional periodic waves in shallow water," J. Fluid Mech. 209, 567-589 (1989).

[21] A. Hasegawa and Y. Kodama, "The guiding-center soliton," Phys. Rev. Lett. 66, 161-164 (1991).

[22] A. Hasegawa and Y. Kodama, "The guiding-center soliton in fibers with periodically varying dispersion," Opt. Lett. 16, 1385-1387 (1991).

[23] A. Hasegawa and Y. Kodama, Solitons in Optical Communications (Oxford University Press, Oxford, 1995).

[24] S. Jin, C. D. Levermore, and D. W. McLaughlin, "The semiclassical limit of the defocusing NLS hierarchy," Commun. Pure Appl. Math. 52, 613-654 (1999).

[25] A. M. Kamchatnov, "New approach to periodic solutions of integrable equations and nonlinear theory of modulational instability," Phys. Rep. 286, 199-270 (1997).

[26] A. M. Kamchatnov, Nonlinear Periodic Waves and Their Modulations: An Introductory Course (World Scientific, New York, 2000).

[27] Y. Kodama, "The Whitham equations for optical communications: Mathematical theory of NRZ," SIAM J. Appl. Math. 59, 2162-2192 (1999).

[28] Y. Kodama and S. Wabnitz, "Analytical theory of guiding-center NRZ and RZ signal transmission in normally dispersive nonlinear optical fibers," Opt. Lett. 20, 2291-2293 (1995).

[29] I. M. Krichever, "Method of averaging for two-dimensional integrable equations," Funct. Anal. Appl. 22, 37-52 (1988).

[30] C. D. Levermore, "The hyperbolic nature of the zero dispersion limit of the KdV equation," Commun. Partial Diff. Eq. 13, 495-514 (1988).

[31] M. V. Pavlov, "Nonlinear Schrödinger equation and the Bogolyubov-Whitham method of averaging," Theor. Math. Phys. 71, 584-588 (1987).

[32] F.-R. Tian, private communication.

[33] F.-R. Tian and J. Ye, "On the Whitham equations for the semiclassical limit of the defocusing nonlinear Schrodinger equation," Commun. Pure Appl. Math. 52, 655-692 (1999).

[34] F.-R. Tian and J. Ye, "On the breaking of the single-phase Whitham solution," Phys. Lett A 277, 115-119 (2000).

[35] G. B. Whitham, "A general approach to linear and nonlinear dispersive waves using a Lagrangian," J. Fluid Mech. 22, 273-283 (1965).

[36] G. B. Whitham, Linear and nonlinear waves (Wiley, New York, 1974).

[37] V. E. Zakharov and A. B. Shabat, "Exact theory of two-dimensional self-focusing and onedimensional self-modulation of waves in nonlinear media," Sov. Phys. JETP 34, 62-69 (1972). 